\title{
REGULARITY OF ORNSTEIN-UHLENBECK PROCESSES DRIVEN BY A LÉVY WHITE NOISE
}

\author{
ZDZISŁAW BRZEŹNIAK AND JERZY ZABCZYK
}

\begin{abstract}
The paper is concerned with spatial and time regularity of solutions to linear stochastic evolution equation perturbed by Lévy white noise "obtained by subordination of a Gaussian white noise". Sufficient conditions for spatial continuity are derived. It is also shown that solutions do not have in general cádlág modifications. General results are applied to equations with fractional Laplacian. Applications to Burgers stochastic equations are considered as well.
\end{abstract}

\section{INTRODUCTION}

The problem of spatial and time regularity of linear stochastic equation with Gaussian noise is well understood, see e.g. [16]. The case of Lévy type perturbations has not been investigated earlier with an exception of the paper [31] by Lescot and Röckner, treating stochastic heat equation with $\alpha$ stable noise.

The present paper is devoted to a systematic study of the spatial and time regularity of the Ornstein-Uhlenbeck process with a general Lévy white noise generalizing the so called stable white noise. Let us recall that a $\mathbb{R}^{d}$ valued Wiener process subordinated by a $\frac{\alpha}{2}$-stable, with $\alpha \in(0,2)$, increasing process is a symmetric $\alpha$-stable process on $\mathbb{R}^{d}$. It is therefore natural to define $\alpha$-stable white noise as a cylindrical Wiener processes on Hilbert spaces subordinated by a real, $\frac{\alpha}{2}$-stable process, with $\alpha \in(0,2)$. For an example see [31]. More generally we define Lévy white noise as a cylindrical Wiener process subordinated by an arbitrary real valued, increasing Lévy process. It can be regarded as an alternative to cylindrical Lévy processes introduced, for instance, in [40], see also [36, 37, 39]. It is difficult to judge at the moment which class will better suit the modelling purposes.

Let $Y$ be a Lévy white noise process on a Hilbert space $H$. Then the OrnsteinUhlenbeck process $X$ driven by $Y$ with generator $A$ is a solution of the so called Langevin equation

$$
\left\{\begin{aligned}
d X(t) & =A X(t) d t+d Y(t), t \geq 0 \\
X(0) & =x
\end{aligned}\right.
$$

Date: October 23, 2015.

Supported by the Polish Ministry of Science and Education project 1PO 3A 03429 "Stochastic evolution equations with Lévy noise". 
where $x \in E$ and $A$ is assumed to be an infinitesimal generator of a $C_{0}$ semigroup $\mathrm{S}=(S(t))_{t \geq 0}$, on a Banach space $E$. In the most interesting cases the semigroup $\mathrm{S}$ is analytic and the space $E$ is a subspace of the space $H$, i.e. $E \subset H$. Moreover,

$$
Y(t)=W(Z(t)), t \geq 0
$$

where $Z$ is a subordinator and $W$ is a cylindrical Wiener process on $H$ that lives on some Banach space $U \supset H$. The latter property is equivalent to the fact the embedding $H \hookrightarrow U$ is $\gamma$-radonifying, see e.g. [4]. Our main aim is to present conditions under which the solutions to (1.1) take values in the space $E$. This type of regularity is of prime interest for the study of nonlinear stochastic equations

$$
d u(t)=(A u(t)+F(u(t)) d t+d Y(t), t \geq 0, u(0)=x,
$$

where $F$ is a nonlinear transformation. In fact if $Y_{A}$ is a solution to (1.1), with $x=0$, then (1.3) can be equivalently written as a stochastic integral equation with random coefficients determined by $Y_{A}$ :

$$
u(t)=S(t) x+\int_{0}^{t} S(t-s) F\left(\left(u(s)+Y_{A}(s)\right) d s, t \geq 0 .\right.
$$

Thus the spatial regularity of $Y_{A}$ determines how irregular the nonlinearity $F$ can be. Of some importance here is also the time regularity of the process. As we will see, the solution to 1.1 does not have, in general, locally bounded trajectories and therefore, to treat nonlinear equations 1.4 it is also necessary to determine when the process $Y_{A}$ has trajectories integrable in some powers.

The paper is organized as follows. In the Preliminaries we recall some basic results on subordination. The nonstandard feature of our presentation is that the subordinated process is a cylindrical one. In the next section we gather results on stochastic integrals of Banach valued functions with respect to Poison random measure. Our main result is Theorem 4.1 from Section 4, formulating sufficient conditions on the semigroup $S$ and on the intensity of the subordinator implying regularity. Various applications are treated in Section 5. A typical example is the following equation

$$
\left\{\begin{aligned}
d X(t) & =-(-\Delta)^{\gamma} X(t)+d Y(t), t \geq 0 \\
X(0) & =0
\end{aligned}\right.
$$

were $\gamma$ is a positive constant, $H=L^{2}(\mathcal{O})$ with $\mathcal{O} \subset \mathbb{R}^{d}$ and $(-\Delta)^{\gamma}$ is the fractional power of the Laplace operator $\Delta$ with the Dirichlet boundary conditions. In particular, if $\gamma=1$, we get stochastic heat equation

$$
\left\{\begin{aligned}
d X(t) & =\Delta X(t)+d Y(t), t \geq 0 \\
X(0) & =0
\end{aligned}\right.
$$


Our paper strengthens considerably a recent result by Lescot and Röckner, who proved in [31, by employing Dirichlet forms and Sazonov Theorem, that if $d=1$, $\gamma=1$, and $Y$ is an $\alpha$ stable process, $\alpha \in(0,2)$, then $X$ takes values in the space $L^{2}(0,1)$, see also comments in subsection 5.4. In fact a special case of our results reads as follows, see Corollaries 5.2 and 5.1 .

Theorem 1.1. Suppose that $\alpha \in(0,2], \gamma \in(0,2]$ and $\delta \in\left[0, \frac{\gamma}{\alpha \vee 1}-\frac{d}{2}\right)$. Then the mild solution $u$ to problem (1.5) is such that for all $t \geq 0$, with probability 1 , $X(t) \in C_{0}^{\delta}(\mathcal{O})$.

The method of the proof is a generalization of a method from [5] (see also [6, Appendix C] for details in the case $d=1$ and $\gamma<1$, where a purely Gaussian case was considered). In that case, i.e. when in equation (1.5) we have purely Gaussian process, i.e. an $L^{2}(0,1)$-cylindrical Wiener process, the mild solution $X$ takes values in the space $C_{0}^{\delta}(0,1)$, for $\delta<\frac{\gamma}{2}-\frac{1}{2}$. Note that here $d=1$ and $\gamma=2$.

Some limits to the spatial regularity is the subject of Section 6 . We consider the Langevin equation (1.6) with the space $L^{2}(0,1)$ replaced by $C(\mathbb{S})$, where $\mathbb{S}$ is the unit circle, and the operator $\Delta$ is replaced by the first order operator $D=\frac{\partial}{\partial \sigma}$. We show that if $Y=f L$ where $L$ is a real valued Lévy process with trajectories of infinite variation then there exists $f \in C(\mathbb{S})$ such that the mild solution of the modified problem (1.6) is not $C(\mathbb{S})$-valued. In this way we generalize a result by Dettweiler and van Neerven [18] from the Gaussian to the Lévy case.

In Section 7 we deal with the time regularity of the trajectories of OrnsteinUhlenbeck processes driven by Lévy white noise and show that trajectories are not locally bounded but have some integrability properties. In Section 8 we establish these properties and apply them to establish an existence result to Burgers equation with Lévy white noise. We discuss only Burgers equation to fix ideas, but we believe that other equations can be treated in a similar way. The Burgers equation with Lévy type noise has been considered in [50], [40] and recently in [20]. Our results are not directly comparable with that of [50] because we deal with different form of noise. The most recent paper [20] considers compound Poisson noise and in fact deals with deterministic Burgers equation on random intervals. A possibility to generalize the results to non-autonomous equations is discussed in the final Section 9.

\section{Preliminaries}

2.1. Subordination. All processes considered in this paper will be defined on a fixed probability space $\mathfrak{P}=(\Omega, \mathcal{F}, \mathbb{P})$. The law of a Banach space valued random 
variable $\xi$ will be denoted by $\mathcal{L}(\xi)$. A family $\left(\mu_{t}\right)_{t \geq 0}$ of a probability measure on a Banach space $U$ is a convolution semigroup if it it satisfies the following properties:

(i) $\mu_{0}=\delta_{0}$,

(ii) $\mu_{t} \rightarrow \mu_{0}$ weakly as $t \searrow 0$,

(iii) $\mu_{t} * \mu_{s}=\mu_{t+s}, t, s \geq 0$.

By definition, a subordinator process, or shortly subordinator, is an increasing Lévy process, see [43, Definitions 1.6 and 21.4]. We will need, see [43, Theorem 21.5], the following fundamental characterization of subordinator processes.

Theorem 2.1. Suppose that $Z=(Z(t))_{t \geq 0}$ is a subordinator process defined on the probability space $\mathfrak{P}=(\Omega, \mathcal{F}, \mathbb{P})$. Then there exists a real number $b \in \mathbb{R}_{+}$, a nonnegative measure $\rho$ on $\left(\mathbb{R}_{+}, \mathcal{B}\left(\mathbb{R}_{+}\right)\right)$satisfying

$$
\rho(\{0\})=0, \int_{1}^{\infty} \rho(d \xi)+\int_{0}^{1} \xi \rho(d \xi)<\infty
$$

such that

$$
\mathbb{E}\left(e^{-r Z(t)}\right)=e^{-t \tilde{\psi}(r)}, r \geq 0, t \geq 0,
$$

where

$$
\tilde{\psi}(r)=b r+\int_{0}^{\infty}\left(1-e^{-r \xi}\right) \rho(d \xi), r \geq 0 .
$$

The family $\left(\mu_{t}\right)_{t \geq 0}$, where $\mu_{t}$ is the laws of $Z(t), t \geq 0$, forms a convolution semigroup on $\mathbb{R}$.

The measure $\rho$, resp. the number $b$, see discussions preceding [43, Def. 11.9], is called the intensity measure, resp. the drift, of the subordinator process $Z$.

Definition 2.2.(i) For $p>0$ we will denote by $\operatorname{Sub}(p)$ the set consisting of all subordinator processes $Z$ whose intensity measure $\rho$ satisfies

$$
\int_{0}^{1} \xi^{\frac{p}{2}} \rho(d \xi)<\infty
$$

It is obvious that if $0<p_{1}<p_{2}<2 \leq p_{3}$, then

$$
\operatorname{Sub}\left(p_{1}\right) \varsubsetneqq \operatorname{Sub}\left(p_{2}\right) \varsubsetneqq \operatorname{Sub}(2)=\operatorname{Sub}\left(p_{3}\right) .
$$

(ii) Note that if $\beta \in(0,1)$ and the measure $\rho$ is defined by

$$
\rho(d \xi)=\frac{1}{\beta \Gamma(1-\beta) \xi^{1+\beta}} 1_{(0, \infty)}(\xi) d \xi,
$$

where $\Gamma$ is the Euler-gamma function $\left(\Gamma(z)=\int_{0}^{\infty} t^{z-1} e^{-t} d t, \operatorname{Re} z>0\right)$, then the measure $\rho$ satisfies condition (2.1). If $b=0$ and $\beta \in(0,1)$, then $\tilde{\psi}=\tilde{\psi}_{\beta}=r^{\beta}, r \geq 0$. 
A subordinator process corresponding to $b=0$ and the measure $\rho$ defined by (2.5) will usually be denoted by $Z^{\beta}$.

Assume now that $\left(\zeta_{s}\right)_{s \geq 0}$ is a convolution semigroup on a Banach space $U$ and $\left(\mu_{s}\right)_{s \geq 0}$ a convolution semigroup on $\mathbb{R}_{+}$corresponding to a subordinator. We define then the subordinated laws on $U$ by

$$
\tilde{\zeta}_{t}:=\int_{0}^{\infty} \zeta_{s} \mu_{t}(d s), t \geq 0
$$

It is easy to check, that the subordinated laws form a convolution semigroup as well:

$$
\tilde{\zeta}_{t+s}=\tilde{\zeta}_{t} * \tilde{\zeta}_{s}, \quad t, s \geq 0 .
$$

If $W$ and $Z$ are independent Lévy processes, with the corresponding convolution semigroups $\left(\zeta_{s}\right)_{s \geq 0}$ and $\left(\mu_{s}\right)_{s \geq 0}$, then the subordinated laws correspond to the Lévy process $Y=(Y(t))_{t \geq 0}$ defined by (1.2) , i.e.

$$
Y(t):=W(Z(t)), t \geq 0 .
$$

We are interested in a special case of the general situation described in the following result.

Theorem 2.3. Suppose that $H$ is a separable Hilbert space and $U$ is a separable Banach space such that $H \subset U$ continuously and densely. Assume that $Z$ is a subordinator process with the intensity measure $\rho$ and the drift $b$. Assume that $W=$ $(W(t))_{t \geq 0}$ is an $U$-valued Wiener process with the RKHS of $W(1)$ equal to $H$ and let $\zeta_{s}=\mathcal{L}(W(s))$.

i) If the process $Y$ is defined by (2.7) then,

$$
\mathbb{E}\left(e^{i\langle Y(t), \phi\rangle_{U, U^{*}}}\right)=e^{-t \tilde{\lambda}(\phi)}, \phi \in U^{*}, t \geq 0 .
$$

where, with $\tilde{\psi}$ defined by (2.3),

$$
\tilde{\lambda}(\phi)=\tilde{\psi}\left(\frac{1}{2}|\phi|_{H}^{2}\right), \phi \in H .
$$

ii) Moreover $Y$ is a $U$-valued Lévy process such that

$$
\mathbb{E}\left(e^{i\langle Y(t), \phi\rangle}\right)=e^{-t \mathrm{PV} \int_{U}\left(1-e^{i\langle u, \phi\rangle}\right) \nu(d u)}, \phi \in U^{*}, t \geq 0,
$$

where the measure $\nu$ is given by formula

$$
\nu(\Gamma)=\int_{0}^{\infty} \zeta_{s}(\Gamma) \rho(d s), \Gamma \in \mathcal{B}(U),
$$

and

$$
\mathrm{PV} \int_{U}\left(1-e^{i\langle u, \phi\rangle}\right) \nu(d u):=\lim _{\varepsilon \searrow 0} \int_{u \in U:|u| \geq \varepsilon}\left(1-e^{i\langle u, \phi\rangle}\right) \nu(d u) .
$$


iii) The process $Y$ is of finite variation iff

$$
\int_{0}^{1}\left[\int_{B_{U}(0,1)}|u|_{U} \zeta_{s}(d u)\right] \rho(d s)<\infty
$$

The process $Y$ will be called an $H$-cylindrical Lévy process subordinated by a (subordinator) process $Z$.

In the identity (2.9),$|\phi|_{H}$ denotes the norm of $\phi$ regarded as a functional on $H$ while in the identity (2.8), the bracket $\langle\cdot, \cdot\rangle_{U, U^{*}}$ is the duality pairing between $U$ and $U^{*}$. Note that the RHS of the equality (2.8) makes sense since we identify $H$ with its dual $H^{\prime}$ so that $U^{*} \subset H^{\prime} \equiv H \subset U$. Several aspects of Theorem 2.3 can be found in [43], where a finite dimensional case is studied, and [32], but we provide a sketch of a proof for the readers convenience.

Proof of Theorem 2.3. First let us observe that the process $Y$ is a well defined $U$ valued cádlág process. For simplicity we will assume that the process $W$, resp. $Z$, is defined on a probability space $\left(\Omega_{1}, \mathcal{F}_{1}, \mathbb{P}_{1}\right)$, resp. $\left(\Omega_{2}, \mathcal{F}_{2}, \mathbb{P}_{2}\right)$ and $\Omega=\Omega_{1} \times \Omega_{2}$, $\mathcal{F}=\mathcal{F}_{1} \otimes \mathcal{F}_{2}$ and $\mathbb{P}=\mathbb{P}_{1} \otimes \mathbb{P}_{2}$. Then, for any $\phi \in H$, we have the following sequence of equalities

$$
\begin{aligned}
\mathbb{E}\left(e_{U, U^{*}}^{i\langle Y(t), \phi\rangle}\right) & =\mathbb{E}_{1} \mathbb{E}_{2}\left(e_{U, U^{*}}^{i\left\langle W\left(Z\left(t, \omega_{2}\right), \omega_{1}\right), \phi\right\rangle}\right) \\
=\mathbb{E}_{2}\left[\mathbb{E}_{1}\left(e_{U, U^{*}}^{i\left\langle W\left(Z\left(t, \omega_{2}\right), \omega_{1}\right), \phi\right\rangle}\right)\right] & =\mathbb{E}_{2} e^{-Z\left(t, \omega_{2}\right) \frac{1}{2}|\phi|^{2}}=e^{-t \tilde{\psi}\left(\frac{1}{2}|\phi|^{2}\right)}=e^{-t \tilde{\lambda}(\phi)} .
\end{aligned}
$$

This concludes the proof of the identity (2.8).

In the first step in proving the identity (2.11) we shall prove that the measure $\nu$ defined by the RHS of equality (2.8) satisfies identity (2.10). By the formulae (2.82.9) it is enough to show that $\phi \in U^{*}, \tilde{\psi}\left(|\phi|_{H}^{2}\right)=\int_{U}\left(1-e^{i\langle u, \phi\rangle}\right) \nu(d u)$. We have, by (2.8) and (2.3), where for simplicity we assume first that $b=0$,

$$
\begin{aligned}
\mathrm{PV} \int_{U}\left(1-e^{i\langle u, \phi\rangle}\right) \nu(d u) & =\int_{0}^{\infty} \mathrm{PV} \int_{U}\left(1-e^{i\langle u, \phi\rangle}\right) \zeta_{s}(d u) \rho(d s) \\
& =\int_{0}^{\infty}\left(1-e^{-\frac{s}{2}|\phi|_{H}^{2}}\right) \rho(d s)=\tilde{\psi}\left(\frac{1}{2}|\phi|_{H}^{2}\right) .
\end{aligned}
$$

This together with the identity (2.10) concludes the proofs of the first two parts. To prove the final part let us recall, see e.g. [40], that an $U$-valued Lévy process $Y$ with intensity measure $\nu$ is of finite variation iff $\int_{B_{U}(0,1)}|u|_{U} \nu(d u)<\infty$. Let now $Y$ and $\nu$ be as in Theorem 2.3. Since then

$$
\int_{B_{U}(0,1)}|u|_{U} \zeta_{s}(d u) \leq \int_{B_{U}(0,1)} \zeta_{s}(d u) \leq \int_{U} \zeta_{s}(d u)=1
$$


and

$$
\begin{gathered}
\int_{B_{U}(0,1)}|u|_{U} \nu(d u)=\int_{0}^{\infty}\left[\int_{B_{U}(0,1)}|u|_{U} \zeta_{s}(d u)\right] \rho(d s)=\int_{0}^{1}\left[\int_{B_{U}(0,1)}|u|_{U} \zeta_{s}(d u)\right] \rho(d s) \\
+\int_{1}^{\infty}\left[\int_{B_{U}(0,1)}|u|_{U} \zeta_{s}(d u)\right] \rho(d s) \leq \int_{0}^{1}\left[\int_{B_{U}(0,1)}|u|_{U} \zeta_{s}(d u)\right] \rho(d s)+\int_{1}^{\infty} \rho(d s),
\end{gathered}
$$

in view of condition (2.1) we infer that $Y$ is of finite variation iff

$$
\int_{0}^{1}\left[\int_{B_{U}(0,1)}|u|_{U} \zeta_{s}(d u)\right] \rho(d s)<\infty
$$

In particular, if $H=U=\mathbb{R}, Y$ is of finite variation iff $\int_{0}^{1} s^{1 / 2} \rho(d s)<\infty$.

Remarks 2.4.(1) Note that since $H$ is the RKHS of $W(1)$, the embedding $H \hookrightarrow U$ is $\gamma$-radonifying.

(2) In view of the Fernique Theorem, $\zeta_{s}$ has finite second moment and moreover there exists $C>0$ such that for all $s>0, \int_{U}|u|^{2} \zeta_{s}(d u) \leq C s$.

(3) Given a separable Hilbert space $H$ and a real number $p \in(0, \infty)$ we will denote by $\operatorname{LSub}(H, p)$, the class of all Lévy processes $Y$ of the form (2.7), where $W$ is $H$ cylindrical Wiener process and $Z$ is an independent subordinator process belonging to class $\operatorname{Sub}(p)$.

(4) In the special case of the subordinator process $Z^{\frac{\alpha}{2}}$, with $\alpha \in(0,2)$, i.e. when $\rho$ is defined by formula (2.5) with $\beta=\frac{\alpha}{2}$, the process $Y$ constructed in Theorem 2.3 will be denoted by $Y^{\alpha}$ and called the $H$-cylindrical $\alpha$-stable process. Note that in this case

$$
\mathbb{E}\left(e^{i\left\langle Y^{\alpha}(t), \phi\right\rangle}\right)=e^{-t \lambda_{\alpha}(\phi)}, \phi \in U^{*}, t \geq 0
$$

where $\lambda_{\alpha}$ is defined by

$$
\lambda_{\alpha}(\phi)=\left(\frac{1}{2}\right)^{\frac{\alpha}{2}}|\phi|_{H}^{\alpha}, \phi \in H
$$

(5) It follows from Definition 2.2(ii) that $Y^{\alpha}$ belongs to the class $\operatorname{LSub}(H, p)$ iff $\alpha<p$.

(6) Let now the Lévy process $Y$ and its intensity measure $\nu$ be as in Theorem 2.3. By part (iii) of that result, the process $Y$ is of finite variation iff $\int_{B_{U}(0,1)}|u|_{U} \nu(d u)<\infty$. Since then

$$
\int_{B_{U}(0,1)}|u|_{U} \zeta_{s}(d u) \leq \int_{B_{U}(0,1)} \zeta_{s}(d u) \leq \int_{U} \zeta_{s}(d u)=1
$$


and

$$
\begin{aligned}
\int_{B_{U}(0,1)}|u|_{U} \nu(d u) & =\int_{0}^{\infty}\left[\int_{B_{U}(0,1)}|u|_{U} \zeta_{s}(d u)\right] \rho(d s) \\
=\int_{0}^{1}\left[\int_{B_{U}(0,1)}|u|_{U} \zeta_{s}(d u)\right] \rho(d s) & +\int_{1}^{\infty}\left[\int_{B_{U}(0,1)}|u|_{U} \zeta_{s}(d u)\right] \rho(d s) \\
\leq \int_{0}^{1}\left[\int_{B_{U}(0,1)}|u|_{U} \zeta_{s}(d u)\right] \rho(d s) & +\int_{1}^{\infty} \rho(d s),
\end{aligned}
$$

in view of condition (2.1) we infer that $Y$ is of finite variation iff

$$
\int_{0}^{1}\left[\int_{B_{U}(0,1)}|u|_{U} \zeta_{s}(d u)\right] \rho(d s)<\infty
$$

In particular, if $H=U=\mathbb{R}$, the process $Y$ is of finite variation iff $\int_{0}^{1} s^{1 / 2} \rho(d s)<\infty$.

(7) It follows from part (5) of these remarks that if in addition $H=U=\mathbb{R}$, the process $Y^{\alpha}$ is of finite variation in $U$ iff $\int_{0}^{1} s^{1 / 2} s^{-1-\frac{\alpha}{2}} d s<\infty$, i.e. iff $\alpha \in(0,1)$.

Remark 2.5. The jump times of the process $Y$ are the same as of the process $Z$.

2.2. Ornstein-Uhlenbeck processes. The main object of our studies are OrnsteinUhlenbeck processes on a Banach space $E$, driven by Lévy white noise $Y$, that is solutions of the Langevin equation:

$$
\left\{\begin{aligned}
d X(t) & =A X(t)+d Y(t), t \geq 0 \\
X(0) & =x \in E
\end{aligned}\right.
$$

Here $E$ is a Banach space such that $E \subset H, A$ is an infinitesimal generator of a $C_{0}$ semigroup on $E, x \in E$ and $Y=(Y(t))_{t \geq 0}$ a Lévy white noise defined by (2.7).

By a mild solution to problem (5.2) we understand an adapted $E$-valued process $X=(X(t))_{t \geq 0}$, such that for each $t \geq 0$, the following holds

$$
X(t)=e^{t A} x+\int_{0}^{t} e^{(t-s) A} d Y(s), t \geq 0 .
$$

As in the theory od stochastic evolution equations driven by a cylindrical Wiener process, see e.g. [11] we can define a notion of a weak solution. In the Hilbert space case the following definition has been proposed by [40], see Definition 9.11. A weak solution of (5.2) is an adapted $E$-valued stochastic process $\{X(t)\}_{t \in \geq 0}$ such that for all $x^{*} \in D\left(A^{*}\right)$ the function $s \mapsto\left\langle X(s), A^{*} x^{*}\right\rangle$ is almost surely locally integrable and

$$
\left\langle X(t), x^{*}\right\rangle=\int_{0}^{t}\left\langle X(s), A^{*} x^{*}\right\rangle d s+\left\langle Y(t), x^{*}\right\rangle \quad t \geq 0 .
$$

Equivalence between various notions of solutions in well known, see e.g. Proposition 4.2 in [4] for the case of strict and mild solutions of equations in Banach spaces driven 
by a Wiener process and Theorem 9.15 in [40] for the case of weak and mild solutions of equations in Hilbert spaces driven by a Lévy noise. In the current work we will not dwell on this issue and we will only consider mild solutions.

The following result is a direct extension to Banach spaces of a result in the Hilbert space case, due to Chojnowska-Michalik, see [15].

Theorem 2.6. If $U$ is a Banach space, $A$ is an infinitesimal generator of a $C_{0}$ semigroup $S=(S(t))_{t \geq 0}$ on $U$ and $Y$ is a $U$-valued process satisfying

$$
\mathbb{E}\left(e^{i\langle Y(t), \phi\rangle}\right)=e^{-t \lambda(\phi)}, \phi \in U^{*},
$$

then the solution $X$ to the following stochastic Langevin equation

$$
\left\{\begin{aligned}
d X(t) & =A X(t) d t+B d Y(t) \\
X(0) & =0
\end{aligned}\right.
$$

satisfies

$$
\mathbb{E}\left(e^{i\langle X(t), \phi\rangle}\right)=e^{-\int_{0}^{t} \lambda\left(B^{*} S^{*}(\sigma) \phi\right) d \sigma}, \quad \phi \in U^{*}
$$

Remark 2.7. Since $X(t)=\int_{0}^{t} S(t-\sigma) B d Y(\sigma)$, equality (2.17) can be equivalently rewritten in terms of the measure $\mu_{t}=\mathcal{L}\left(X_{t}\right)$ as follows

$$
\hat{\mu}_{t}(\phi)=e^{-\int_{0}^{t} \lambda\left(B^{*} S^{*}(\sigma) \phi\right) d \sigma}, \phi \in U^{*} .
$$

Proof of Theorem 2.6. Denote $\Psi(\sigma):=S(t-\sigma) B$. By the assumption (2.15) and using approximations of Riemann sums we have

$$
\begin{aligned}
\mathbb{E} e^{\left\langle\xi, \int_{0}^{t} \Psi(\sigma) d Y(\sigma)\right\rangle} & =\lim _{N} \mathbb{E} e^{\left\langle\xi, \sum_{k} \Psi\left(\sigma_{k}\right)\left(Y\left(\sigma_{k+1}\right)-Y\left(\sigma_{k}\right)\right)\right\rangle} \\
=\lim _{N} \prod_{k} \mathbb{E} e^{\left\langle\Psi\left(\sigma_{k}\right)^{*} \xi,\left(Y\left(\sigma_{k+1}\right)-Y\left(\sigma_{k}\right)\right)\right\rangle} & =\lim _{N} \prod_{k} e^{-\left(\sigma_{k+1}-\sigma_{k}\right) \lambda\left(\Psi\left(\sigma_{k}\right)^{*} \xi\right)} \\
=e^{-\lim _{N} \sum_{k}\left(\sigma_{k+1}-\sigma_{k}\right) \lambda\left(\Psi\left(\sigma_{k}\right)^{*} \xi\right)} & =e^{-\int_{0}^{t} \lambda\left(\Psi^{*}(\sigma) \phi\right) d \sigma} .
\end{aligned}
$$

\section{Integrals With Respect to Poisson Random measures}

We will need several results on stochastic integration of Banach space valued functions with respect to Poisson random measure. Suppose that $\pi$ is a Poisson random measure on a measure space $(G, \mathcal{G})$ with intensity $\nu_{\pi}$ over the probability space $\mathfrak{P}=(\Omega, \mathcal{F}, \mathbb{P})$. Suppose also that $E$ is a separable Banach space. 
Theorem 3.1. Suppose that $p \in(0,1]$. Then for an arbitrary strongly measurable function $f: G \rightarrow E$, belonging to $L^{p}\left(G, \mathcal{G} ; \nu_{\pi} ; E\right)$, the following inequality holds

$$
\mathbb{E}\left|\int f(x) \pi(d x)\right|_{E}^{p} \leq \int|f(x)|^{p} \nu_{\pi}(d x) .
$$

Proof of Theorem 3.1. Let us fix $p \in(0,1]$. Since the space of finitely-valued functions is dense in $L^{p}(G, \mathcal{G} ; \nu ; E)$, see Lemma 1.1. in [16], we may suppose that $f=\sum_{i} f_{i} 1_{B_{i}}$ with $f_{i} \in E$, and $B_{i} \in \mathcal{G}$ is finite family of pair-wise disjoint sets such that $\nu_{\pi}\left(B_{i}\right)<\infty$. Then

$$
\int_{G} f(x) \pi(d x)=\sum_{i} \pi\left(B_{i}\right) f_{i}
$$

Moreover, as $\pi\left(B_{i}\right)$ takes values in $\mathbb{N}, \mathbb{E}\left|\pi\left(B_{i}\right)\right|^{p} \leq \mathbb{E}\left|\pi\left(B_{i}\right)\right|$. Since the random variables $\pi\left(B_{i}\right)$ are independent, we infer that

$$
\begin{aligned}
\mathbb{E}\left|\int_{G} \xi(x) \pi(d x)\right|_{E}^{p} & =\mathbb{E}\left[\left|\sum_{i} \pi\left(B_{i}\right) f_{i}\right|_{E}^{p}\right] \\
& \leq \sum_{i}\left|f_{i}\right|_{E}^{p} \mathbb{E}\left|\pi\left(B_{i}\right)\right|^{p} \leq \sum_{i}\left|f_{i}\right|_{E}^{p} \mathbb{E}\left|\pi\left(B_{i}\right)\right| \\
& \leq \sum_{i}\left|f_{i}\right|_{E}^{p} \nu_{\pi}\left(B_{i}\right)=\mathbb{E} \int_{G}|f(x)|_{E}^{p} \nu_{\pi}(d x) .
\end{aligned}
$$

The proof is complete.

If $p \in(1,2]$, we will need that $E$ is o type $p$. Let us recall that a Banach space $E$ is is a type $p$, with $p \in[1,2]$, iff there exists a constant $K_{p}(E)>0$ for any finite sequence $\varepsilon_{1}, \ldots, \varepsilon_{n}: \Omega \rightarrow\{-1,1\}$ of symmetric i.i.d. random variables and for any finite sequence $x_{1}, \ldots, x_{n}$ of elements of $X$, the following inequality holds

$$
\mathbb{E}\left|\sum_{i=1}^{n} \varepsilon_{i} x_{i}\right|^{p} \leq K_{p}(X) \sum_{i=1}^{n}\left|x_{i}\right|^{p} .
$$

Remark 3.2. It is well known that all $L^{q}$ spaces with $q \geq p$ are type $p$ Banach spaces. Moreover, see e.g. Corollary A.6 in [4], the classical Sobolev $H^{s, q}$ and BesovSlobodetski spaces $W^{s, q}$, with $q \geq p$, are also type $p$ Banach spaces.

We have the the following extension of Lemma 7 from [8].

Theorem 3.3. Suppose that $p \in(1,2]$ and $E$ is a type $p$ Banach space. Then for an arbitrary strongly measurable function $f: G \rightarrow E$, from $L^{p}(G, \mathcal{G} ; \nu ; E)$, the following inequality holds

$$
\mathbb{E}\left|\int_{G} f(x) \tilde{\pi}(d x)\right|_{E}^{p} \leq 2^{2-p} K_{p}(E) \int_{G}|f(x)|^{p} \nu_{\pi}(d x),
$$

where $\tilde{\pi}$ is the compensated random measure defined by $\tilde{\pi}(B)=\pi(B)-\nu_{\pi}(B), B \in \mathcal{G}$. 
Proof of Theorem 3.3. The following equivalent characterization of type $p$ Banach spaces holds, see [26, Theorem 2.1]:

A Banach space $X$ is is of type $p$ iff there exists $K_{p}(X)$ such that for any finite sequence $\xi_{1}, \ldots, \xi_{n}$ of independent $X$-valued random variables mean zero with finite p-th moments,

$$
\mathbb{E}\left|\sum_{i=1}^{n} \xi_{i}\right|^{p} \leq K_{p}(X) \sum_{i=1}^{n} \mathbb{E}\left|\xi_{i}\right|^{p} .
$$

As in the proof of Theorem 3.1 we may assume that $f=\sum_{i} f_{i} 1_{B_{i}}$ with $f_{i} \in E$, and $B_{i} \in \mathcal{G}$ is finite family of pair-wise disjoint sets such that $\nu_{\pi}\left(B_{i}\right)<\infty$. Since then

$$
\int_{G} f(x) \tilde{\pi}(d x)=\sum_{i} \tilde{\pi}\left(B_{i}\right) f_{i}
$$

and the random variables $\tilde{\pi}\left(B_{i}\right)$ are independent and of mean 0 , by the just quoted characterization of the type $p$ property of the Banach space $E$ we infer that

$$
\begin{aligned}
\mathbb{E}\left|\int_{G} \xi(x) \tilde{\pi}(d x)\right|_{E}^{p} & =\mathbb{E}\left[\left|\sum_{i} \tilde{\pi}\left(B_{i}\right) f_{i}\right|_{E}^{p}\right] \\
& \leq K_{p}(E) \sum_{i}\left|f_{i}\right|_{E}^{p} \mathbb{E}\left|\tilde{\pi}\left(B_{i}\right)\right|^{p} \leq K_{p}(E) 2^{2-p} \sum_{i}\left|f_{i}\right|_{E}^{p} \mathbb{E}\left|\tilde{\pi}\left(B_{i}\right)\right| \\
& \leq K_{p}(E) 2^{2-p} \sum_{i}\left|f_{i}\right|_{E}^{p} \nu_{\pi}\left(B_{i}\right)=K_{p}(E) 2^{2-p} \mathbb{E} \int_{G}|f(x)|_{E}^{p} \nu_{\pi}(d x) .
\end{aligned}
$$

In the above we have used a fact, see Lemma 6 in [8], that if $\xi \sim$ Poiss $(\lambda)$, with $\lambda>0$ and $p \in(1,2], \mathbb{E}|\xi-\lambda|^{p} \leq 2^{2-p} \lambda$. The proof is complete.

\section{Space Regularity of the Ornstein-Uhlenbeck Process}

We consider a triple of spaces $E, H$ and $U$ such that the middle one is a Hilbert space and the other two are separable Banach spaces and

$$
E \subset H \subset U
$$

We consider an $U$-valued Wiener process $W=(W(t))_{t \geq 0}$ (with RKHS $H$ ) and a subordinator process $Z=(Z(t))_{t \geq 0}$. As in Theorem 2.3 we define an $U$-valued Lévy process $Y=(Y(t))_{t \geq 0}$ by the subordination formula (1.2) , i.e.

$$
Y(t):=W(Z(t)), t \geq 0 .
$$

We also consider a $C_{0}$ semigroup $\mathrm{S}=(S(t))_{t \geq 0}$ of bounded linear operators on $E$. Our aim is to study spatial regularity of Ornstein-Uhlenbeck processes driven 
by the Lévy process $Y$ with generator $A$. In other words we intend to study spatial regularity of a process $X$ which is a solution of the Langevin equation (1.1), i.e.

$$
\left\{\begin{aligned}
d X(t) & =A X(t) d t+d Y(t), t \geq 0 \\
X(0) & =0
\end{aligned}\right.
$$

However we begin with the following preliminary result.

Theorem 4.1. Assume that $T>0, p \in(0,2]$ and $\Psi(t), t \in[0, T]$, is a strongly measurable function with values in the space of all linear and bounded operators from $U$ to E such that

$$
\int_{0}^{T}|\Psi(s)|_{L(U, E)}^{p} d s<\infty .
$$

Assume that $Z$ is a subordinator process belonging to the class $\operatorname{Sub}(p)$. Let $Y$ be the $U$ valued Lévy process defined by formula (4.2). Then, with probability 1, $\int_{0}^{t} \Psi(s) d Y(s)$ takes values in $E$, for all $t \in[0, T]$, if

(i) $p \in(0,1]$,

(ii) or $p \in(1,2]$ and the Banach space $E$ is of type $p$.

Remark 4.2. (i) Let us notice that if the condition (4.4) is satisfied and the embedding $i: H \hookrightarrow U$ is $\gamma$-radonifying, then with $\|\cdot\|_{R(H, E)}^{p}$ denoting the $\gamma$-radonifying norm, see e.g. [5],

$$
\int_{0}^{T}\|\Psi(s)\|_{R(H, E)}^{p} d s<\infty .
$$

The case when $E$ is a Hilbert space and $p=2$ is studied in [40].

(ii) Let us notice that if the condition (4.4) is satisfied and $p_{2}<p$, then it is also satisfied for $p_{2}$.

Proof of Theorem 4.1. The Lévy process $Y$ can be decomposed into two parts, the first one with small jumps and the second one with large jumps:

$$
Y(t)=Y_{1}(t)+Y_{2}(t), t \geq 0
$$

The processes $Y_{1}$ and $Y_{2}$ are defined as follows. Let $\nu$ denotes the intensity measure corresponding to the process $Y$. Then $Y_{1}$ is the Lévy process corresponding to the intensity measure $\nu_{1}$ defined by

$$
\nu_{1}(\Gamma):=\nu\left(\Gamma \cap B_{U}(0,1),\right.
$$

where $B_{U}(0,1)$ is the closed unit ball in $U$. We also define $Y_{2}$ to be the Lévy process corresponding to the intensity measure $\nu_{2}=\nu-\nu_{1}$. Thus $Y_{2}$ is a compound Poisson 
process with the intensity measure $\nu_{2}$. The processes $Y_{1}$ and $Y_{2}$ can be easily constructed in terms of the Poisson random measure $\pi$ associated with the process $Y$ defined by

$$
\pi([0, t] \times \Gamma):=\sum_{s \leq t} 1_{\Gamma}(\Delta Y(s)), \Gamma \in \mathcal{U},
$$

where $\Delta Y(s):=Y\left(s^{+}\right)-Y\left(s^{-}\right), s \geq 0$. Note that we assume the process $Y$ to be right-continuous with left hand limits, so that the definition of $\Delta Y$ makes sense and $Y\left(s^{+}\right)=Y(s)$. It can be shown, see e.g. [27, Prop. 15.5] that $\pi$ is a time homogenous Poisson random measure and that $Y$ can be expresses in terms of the random measure $\pi$ as follows

$$
Y(t)=\sum_{s \leq t} \Delta Y(s)=\int_{0}^{t} \int_{U} u \pi(d y, d s)
$$

Then we define processes $Y_{1}$ and $Y_{2}$ by the following modifications of the previous formula.

$$
\begin{aligned}
& Y_{2}(t)=\sum_{s \leq t} 1_{\{|\Delta Y(s)| \geq 1\}} \Delta Y(s)=\int_{0}^{t} \int_{|u| \geq 1} u \pi(d y, d s) \\
& Y_{1}(t)=\sum_{s \leq t} 1_{\{|\Delta Y(s)|<1\}} \Delta Y(s)=\int_{0}^{t} \int_{|u|<1} u \pi(d y, d s)
\end{aligned}
$$

Thus

$$
\int_{0}^{t} \Psi(s) d Y(s)=\int_{0}^{t} \Psi(s) d Y_{1}(s)+\int_{0}^{t} \Psi(s) d Y_{2}(s)
$$

and we will check that the integrals with respect to $Y_{1}$ and $Y_{2}$ take values in $E$.

The process $Y_{2}$ has a discrete sequence of jump times: $0<\tau_{1}<\tau_{2}<\tau_{3}<\cdots$ diverging to $+\infty$, and $\Delta Y_{2}\left(\tau_{k}\right)=\Delta Y\left(\tau_{k}\right)$ for all $k$. Hence the integral with respect to $Y_{2}$ is defined as

$$
\int_{0}^{t} \Psi(s) d Y_{2}(s):=\sum_{\tau_{k} \leq t} \Psi\left(\tau_{k}\right) \Delta Y_{2}\left(\tau_{k}\right) .
$$

Since the operators $\Psi$ take values in $E$ and the sum in (4.11) is finite, it is clear that the integral with respect to the process $Y_{2}$ takes values in $E$.

We pass now to the integral with respect to the process $Y_{1}$ and assume that $p \in$ $(0,1]$. Then, by Lemma 3.1.

$$
\begin{aligned}
\mathbb{E}\left|\int_{0}^{t} \Psi(r) d Y_{1}(r)\right|_{E}^{p} & \leq \int_{0}^{t} \int_{B_{U}(0,1)}|\Psi(r)|_{\mathcal{L}(U, E)}^{p}|u|_{U}^{p} \nu(d u) d r \\
& =\int_{0}^{t}|\Psi(r)|_{\mathcal{L}(U, E)}^{p} d r \int_{B_{U}(0,1)}|u|_{U}^{p} \nu(d u) .
\end{aligned}
$$


It follows directly from formula (2.11) in Theorem 2.3 that for any $p \in(0, \infty)$,

$$
\int_{B_{U}(0,1)}|u|_{U}^{p} \nu(d u)=\int_{0}^{\infty}\left[\int_{B_{U}(0,1)}|u|_{U}^{p} \zeta_{s}(d u)\right] \rho(d s)
$$

We have the following elementary lemma.

Lemma 4.3. If $p \in(0,2]$, then or an arbitrary subordinator process $Z$ from the class $\operatorname{Sub}(p)$, there exists a constant $C>0$ such that

$$
\begin{aligned}
\int_{1}^{\infty}\left[\int_{B_{U}(0,1)}|u|_{U}^{p} \zeta_{s}(d u)\right] \rho(d s) & \leq \int_{1}^{\infty} \rho(d s), \\
\int_{0}^{1} \int_{B_{U}(0,1)}|u|_{U}^{p} \zeta_{s}(d u) \rho(d s) & \leq C \int_{0}^{1} s^{\frac{p}{2}} \rho(d s),
\end{aligned}
$$

where $\nu$ is the intensity measure of $Z$. In particular,

$$
\int_{B_{U}(0,1)}|u|_{U}^{p} \nu(d u) \leq \int_{1}^{\infty} \rho(d s)+C \int_{0}^{1} s^{\frac{p}{2}} \rho(d s) .
$$

Proof of Lemma 4.3. To prove the first inequality we note that

$$
\begin{aligned}
\int_{1}^{\infty}\left[\int_{B_{U}(0,1)}|u|_{U}^{p} \zeta_{s}(d u)\right] \rho(d s) & \leq \int_{1}^{\infty} \int_{B_{U}(0,1)}|u|_{U}^{p} \zeta_{s}(d u) \rho(d s) \\
\leq \int_{1}^{\infty} \zeta_{s}\left(B_{U}(0,1)\right) \rho(d s) & \leq \int_{1}^{\infty} \rho(d s)<\infty .
\end{aligned}
$$

To prove the second inequality we note that by the Cauchy-Schwartz inequality and part (3) of Remark 2.4,

$$
\begin{aligned}
\text { LHS } & \leq \int_{0}^{1} \int_{U}|u|_{U}^{p} \zeta_{s}(d u) \rho(d s) \\
& \leq \int_{0}^{1}\left(\int_{U}|u|_{U}^{2} \zeta_{s}(d u)\right)^{\frac{p}{2}} \rho(d s) \leq \int_{0}^{1}(C s)^{\frac{p}{2}} \rho(d s)=C \int_{0}^{1} s^{\frac{p}{2}} \rho(d s) .
\end{aligned}
$$

By the above Lemma the case $p \in(0,1]$ of Theorem 4.1 follows.

To treat the case of $p \in(1,2]$ note that he integral with respect to $Y_{1}$ is defined as

$$
\begin{aligned}
\int_{0}^{t} \Psi(s) d Y_{1}(s) & :=\int_{0}^{t} \int_{|u|<1} \Psi(s) u \pi(d u, d s) \\
& =\int_{0}^{t} \int_{|u|<1} \Psi(s) u \tilde{\pi}(d u, d s)+\mathrm{PV} \int_{0}^{t} \int_{|u|<1} \Psi(s) u \nu(d u) d s
\end{aligned}
$$

Since PV $\int_{0}^{t} \int_{|u|<1} \Psi(s) u \nu(d u) d s=0$, see e.g. [27, Thm 15.5] we infer that

$$
\int_{0}^{t} \Psi(s) d Y_{1}(s)=\int_{0}^{t} \int_{|u|<1} \Psi(s) u \tilde{\pi}(d u, d s) .
$$


Since we assume that the space $E$ is martingale type $p$, by Lemma 3.3, there exists a constant $C=C_{p}>0$ such that

$$
\mathbb{E}\left|\int_{0}^{t} \int_{B_{U}(0,1)} \Psi(r) u \tilde{\pi}(d u, d r)\right|_{E}^{p} \leq C \int_{0}^{t} \int_{B_{U}(0,1)}|\Psi(r) u|_{E}^{p} \nu(d u) d r .
$$

Therefore there exists a constant $C^{\prime}$ such that

$$
\begin{aligned}
\mathbb{E}\left|\int_{0}^{t} \Psi(r) d Y_{1}(r)\right|_{E}^{p} & \leq C \int_{0}^{t} \int_{B_{U}(0,1)}|\Psi(r) u|_{E}^{p} \nu(d u) d r \\
& \leq C^{\prime} \int_{0}^{t}|\Psi(r)|_{L(U, E)}^{p} d r \int_{B_{U}(0,1)}|u|_{E}^{p} \nu(d u) d r .
\end{aligned}
$$

Taking into account Lemma 4.3, the result follows.

Now we formulate the following important and useful consequence of Theorem 4.1 .

Theorem 4.4. Assume that $p \in(0,2], E$ is a Banach space, $Z$ is a subordinator process belonging to the class $\operatorname{Sub}(p)$ and $Y$ is the Lévy process defined by formula (4.2). Assume that $S=(S(t))_{t \geq 0}$ is $C_{0}$ semigroup on $E$ such that for each $t>0$, $S(t)$ is a bounded and linear operator from $U$ to $E$. Furthermore, we assume that there exists $\theta>0$ with $\theta p<1$ such that and for each $T>0$ one can find $C>0$ such that

$$
|S(r)|_{\mathcal{L}(U, E)} \leq C r^{-\theta}, 0<r \leq T .
$$

Then for all $t \in \mathbb{R}_{+}$, with probability $1, \int_{0}^{t} S(t-r) d Y(r)$ takes values in $E$ provided $p \in(0,1]$ or $p \in(1,2]$ and $E$ is of type $p$.

Proof. It is enough to prove the Theorem for $t \in[0, T]$ for any fixed $T>0$. But then the condition (4.18) implies the condition (4.4) is satisfied with $p<\frac{1}{\theta}$ by a family $\Psi(r)=S(T-r), r \in(0, T]$. Hence the result follows from 4.1 .

Remark 4.5. (i) As observed in the proof above, condition (4.18) implies (4.4) for $p<\frac{1}{\theta}$. Hence we see the advantage of being able to treat the $p \leq 1$ case.

(ii) If $\rho(d s)=s^{-1-\beta}$, then the condition (2.4) is satisfied iff $p>\beta$. Hence, if $\beta$ is fixed there is a lower limit on useful $p$.

(iii) If $\rho(d s)=s^{-1-\beta}$, the condition (4.18) is satisfied and $\beta<\frac{1}{\theta}$, then part (i) of Theorem 4.1 is applicable with any $p \in\left(\beta, \frac{1}{\theta}\right)$. However, if $\beta \geq \frac{1}{\theta}$, then we need a different method. Such a method is proposed in part (ii) of Theorem 4.1. (iv) If $E=U$ and $\mathrm{S}=(S(t))_{t \geq 0}$ is a $C_{0}$ semigroup on $E$, then the condition (4.4) is satisfied for $p \in(0,2]$ and hence Theorem above holds true. In particular, if $A$ denotes the generator of the semigroup $\mathrm{S}$, then, provided that $p \in(0,1]$ or $p \in(1,2]$ and $E$ is a martingale type $p$ Banach space, for each $t>0, X(t) \in E$ a.s. where $X$ is a solution 
of (1.1). Moreover, if $S=(S(t))_{t \geq 0}$ is a $C_{0}$ group on a martingale type $p$ Banach space $E, p \in(1,2]$, then the solution $X$ is $E$-valued cádlág. Indeed, as in [24, 25], we have

$$
X(t)=\int_{0}^{t} S(t-r) d Y(r)=S(t) \int_{0}^{t} S(-r) d Y(r), t \geq 0 .
$$

Since by [8], the process $\int_{0}^{t} S(-r) d Y(r), t \geq$ is $E$-valued cádlág, we infer that the process $X$ is also $E$-valued cádlág.

\section{Spatial Regularity of OU processes With fractional Laplacian}

In this section we investigate spatial regularity of solutions $X$ of the Langevin equations (1.5),

$$
\left\{\begin{aligned}
d X(t) & +(-\Delta)^{\gamma} X(t)=d Y(t), t \geq 0 \\
X(0) & =0
\end{aligned}\right.
$$

where $(-\Delta)^{\gamma}, \gamma>0$, is a fractional Laplacian, with Dirichlet boundary conditions on a set $\mathcal{O} \subset \mathbb{R}^{d}$ and $Y$ is a Lévy white noise in $L^{2}(\mathcal{O})$. Our main aim is find conditions on the various parameters guaranteeing that the solution $X$ takes values in the space $E=C_{0}(\mathcal{O})$. We will treat the equation as an abstract problem on a space $E$,

$$
\left\{\begin{aligned}
d X(t) & =A X(t)+d Y(t), t \geq 0, \\
X(0) & =0
\end{aligned}\right.
$$

with $E$ the Banach space such that $E \subset H, A$ is an infinitesimal generator of a $C_{0}$ semigroup on $E, x \in E$ and $Y=(Y(t))_{t \geq 0}$ a Lévy white noise defined by (2.7). We can regard Laplacian $\Delta$, on $\mathcal{O}$ and with zero boundary conditions, as a self-adjoint operator and the infinitesimal generator of a strongly continuous semigroup of selfadjoint operators $T(t)$ on $L^{2}(\mathcal{O})$. The semigroup can be restricted or extended to $L^{q}(\mathcal{O})$ in a natural way. In this and similar situations the extensions or restrictions will often be denoted in the same way as the original objects.

Our main tool in the study will be Theorem 4.1 and the validity of condition (4.18). We will have also to check that the space $U$ is chosen in such a way, that the process $Y$ is $U$-valued, or equivalently that the embedding $H \hookrightarrow U$ is $\gamma$-radonifying.

5.1. Analytic preliminaries. Let us assume that $\mathcal{O} \subset \mathbb{R}^{d}$ is a bounded open set with sufficiently smooth boundary. We will consider a real number $q$ such that $q \in(1, \infty)$.

By $H^{k, q}(\mathcal{O})$, for $k \in \mathbb{N}, q \in[1, \infty)$ we denote the Banach space of all $f \in L^{q}(\mathcal{O})$ such that $D^{\alpha} f \in L^{q}(\mathcal{O})$, if the multi-index $\alpha \in \mathbb{N}^{d}$ is of length $\leq k$. The norm in 
$H^{k, q}(\mathcal{O})$ is given by

$$
\|f\|_{H^{k, q(\mathcal{O})}}=\left(\sum_{|\alpha| \leq k}\left|D^{\alpha} f\right|_{L^{q}(\mathcal{O})}^{q}\right)^{\frac{d}{q}} .
$$

We define the fractional order Sobolev space $H^{\beta, q}(\mathcal{O}), \beta \in \mathbb{R}_{+} \backslash \mathbb{N}$ by the complex interpolation method, see [34, i.e.

$$
H^{\beta, q}(\mathcal{O})=\left[H^{k, q}(\mathcal{O}), H^{m, q}(\mathcal{O})\right]_{\theta},
$$

where $k, m \in \mathbb{N}, \theta \in(0,1), k<m$, are chosen to satisfy

$$
\beta=(1-\theta) k+\theta m \text {. }
$$

One should bear in mind that the space on the LHS of formula (5.4) does not depend on $k, m, \theta$ provided they satisfy condition (5.5).

In what follows by $H_{0}^{s, q}(\mathcal{O}), s \geq 0, q \in(1, \infty)$ we will denote the closure of $C_{0}^{\infty}(\mathcal{O})$ in the Banach space $H^{s, q}(\mathcal{O})$. It is well known, see e.g. [33, Theorem 11.1], [49, Theorem 1.4.3.2 p.317] and [35, Theorem 3.40] that $H_{0}^{s, q}(\mathcal{O})=H^{s, q}(\mathcal{O})$ iff $s \leq \frac{1}{q}$.

Remark 5.1. It is well known, see e.g. [41] and [4, Appendix B] that all $L^{q}$ spaces, $q \geq p$, are of type $p$. By interpolation, $H_{0}^{s, q}$ and $H_{0}^{s, q}$ spaces, $q \geq p$, are also of type $p$, see e.g. [4, Appendix A] for an argument used in the case of martingale type 2 spaces but which works also in the present situation.

Let us denote by $A_{q}$ the Laplace operator in the space $L^{q}(\mathcal{O})$, with the Dirichlet boundary conditions, i.e. $D\left(A_{q}\right)=H^{2, q} \cap H_{0}^{1, q}$ and $A_{q} u=\Delta u$, for $u \in D\left(A_{q}\right)$.

It is well known, see e.g. [48], that $-A_{q}$ is an operator with bounded imaginary powers and in particular the operator $A_{q}^{\gamma / 2}:=-\left(-A_{q}\right)^{\gamma / 2}$, for $\gamma \in(0,2)$, is a generator of an analytic semigroup on $L^{q}(\mathcal{O})$. Moreover, it follows from Theorem 1.15.3 on p. 103 in [49] that for $\gamma \in(0,2), D\left(A_{q}^{\gamma / 2}\right)=D\left(\left(-A_{q}\right)^{\gamma / 2}\right)=\left[L^{q}(\mathcal{O}), D\left(A_{q}\right)\right]_{\gamma / 2}$, where $\left[L^{q}(\mathcal{O}), D\left(A_{q}\right)\right]_{\gamma / 2}$ is the complex interpolation space of order $\gamma / 2$, see e.g. [33, [49] and [47, Theorem 4.2]. Moreover, by Seeley [44, see also the monograph [49, Theorem in section 4.4.3], we have that for $q \in(1, \infty)$,

$$
D\left(A_{q}^{\gamma / 2}\right)= \begin{cases}H^{\gamma, q}(\mathcal{O}) \cap H_{0}^{1, q}(\mathcal{O}), & \text { if } 1<\gamma \leq 2, \\ H_{0}^{\gamma, q}(\mathcal{O}), & \text { if } \frac{1}{q}<\gamma \leq 1, \\ H^{\gamma, q}(\mathcal{O}), & \text { if } \gamma<\frac{1}{q}\end{cases}
$$

Proposition 5.2. Assume that $H=L^{2}(\mathcal{O})$ and $U=H^{-s, q}(\mathcal{O})$ with

$$
s>\frac{d}{2}, q \in[1, \infty) \text {. }
$$


i) If

$$
r>0, r+s<\gamma
$$

then the $C_{0}$ semigroup $\left(e^{t A_{q}^{\gamma / 2}}\right)_{t \geq 0}$ satisfies condition (4.18) with exponent $\theta:=\frac{r+s}{\gamma}$, $E=H_{0}^{r, q}(\mathcal{O})$ and $U=H^{-s, q}(\mathcal{O})$,

ii) If

$$
\delta+\frac{d}{q}<\gamma-\frac{d}{2}, \delta \geq 0
$$

then the semigroup $\left(e^{t A_{q}^{\gamma / 2}}\right)_{t \geq 0}$ satisfies condition (4.18) with $E=C_{0}^{\delta}(\mathcal{O}), U=$ $H^{-s, q}(\mathcal{O})$ and the exponent $\theta \geq \frac{\delta+\frac{d}{2}}{\gamma}$.

Proof of Proposition 5.2. To prove the first statement note that for $\gamma<\frac{1}{q}, H_{0}^{\gamma, q}(\mathcal{O})=$ $H^{\gamma, q}(\mathcal{O})$. Let us denote by $S=S_{q, \gamma}$, the semigroup on the Banach space $L^{q}(\mathcal{O})$ generated by the operator $A_{q}^{\gamma / 2}$, i.e. $S_{q, \gamma}(t)=e^{t A_{q}^{\gamma / 2}}, t \geq 0$. It follows from (5.6) and some standard estimates on the norm of the analytic semigroup between domains of fractional powers of the generator, see e.g. [38], that there exists a constant $C>0$ such that

$$
\begin{aligned}
\left|S_{q, \gamma}(t)\right|_{L\left(H^{-s, q}, H_{0}^{r, q}\right)} & \leq C\left|S_{q, \gamma}(t)\right|_{\mathcal{L}\left(D\left(A_{q}^{-\frac{s}{\gamma}}\right), D\left(A_{q}^{\frac{r}{\gamma}}\right)\right)} \\
& \leq C t^{-\frac{s}{\gamma}-\frac{r}{\gamma}}=C t^{-\frac{r+s}{\gamma}}
\end{aligned}
$$

Thus the first statement is a direct consequence of inequality (5.10).

To prove the second part of the Proposition let us put $\varepsilon:=\gamma-\frac{d}{2}-\left(\delta+\frac{d}{q}\right)$ which, in view of assumption (5.9), is $>0$. Let us choose a numbers $r$ such that

$$
r>\delta+\frac{d}{q}
$$

and $r-\left(\delta+\frac{d}{q}\right)<\frac{\varepsilon}{2}$ and a number $s$ satisfying the condition (5.7) such that $s-\frac{d}{2}<\frac{\varepsilon}{2}$. Then $H_{0}^{r, q}(\mathcal{O}) \subset E:=C_{0}^{\delta}(\mathcal{O})$ and by inequality (5.10) we infer that

$$
\left|S_{q, \gamma}(t)\right|_{L(U, E)} \leq C t^{-\frac{r+s}{\gamma}} .
$$

Since $r+s<\left(\delta+\frac{d}{q}\right)+\frac{\varepsilon}{2}+\frac{d}{2}+\frac{\varepsilon}{2}=\delta+\frac{d}{q}+\frac{d}{q}+\varepsilon=\gamma$ we infer that condition (4.18) is satisfied with $\theta=\frac{\delta+\frac{d}{2}}{\gamma}$. It can easily shown that condition (4.18) is satisfied with any $\theta>\frac{\delta+\frac{d}{2}}{\gamma}$.

Remark 5.3. In fact we can replace the space $H=L^{2}(\mathcal{O})$ by $H^{\vartheta, 2}(\mathcal{O})$ with, for concreteness, $\vartheta \in\left(-\frac{1}{2}, \frac{1}{2}\right)$. Let us recall that for $\theta \in\left[0, \frac{1}{2}\right), H^{\vartheta, 2}(\mathcal{O})=H_{0}^{\vartheta, 2}(\mathcal{O})$, i.e. the space $C_{0}^{\infty}(\mathcal{O})$ is dense in $H^{\vartheta, 2}(\mathcal{O})$. Then an appropriate version of Proposition 
5.2 holds true. Let us formulate, for future reference, the first two parts.

Let $H=H^{\vartheta, 2}(\mathcal{O})$ with $\vartheta \in\left(-\frac{1}{2}, \frac{1}{2}\right)$ and $U=H^{-s, q}(\mathcal{O})$.

i) If

$$
s>\frac{d}{2}-\vartheta, \quad q \in[1, \infty)
$$

then the embedding $H \hookrightarrow U$ is $\gamma$-radonifying.

ii) If (5.13) holds and

$$
r>0, r+s<\gamma
$$

then, the family $\Psi=S_{q, \gamma}(\cdot)$ satisfies condition (4.18) with exponent $\theta:=\frac{r+s}{\gamma}$ and $E=H_{0}^{r, q}(\mathcal{O})$.

Let us also note that the supremum of all numbers $r$ satisfying conditions 5.13 5.14) is equal to $\gamma+\vartheta-\frac{d}{2}$.

5.2. OU process driven by a cylindrical $\alpha$-stable processes with $\alpha<1$. The following result is a direct consequence of Theorem 4.1 part (i) and Proposition 5.2. Let us recall that by $\operatorname{LSub}\left(L^{2}, p\right)$ we denote the class of all cylindrical Lévy processes defined by formula (2.7) with $W$ being a cylindrical Wiener process on the Hilbert space $H=L^{2}$ and $Z$ being a subordinator process belonging to the class $\operatorname{Sub}(p)$.

Theorem 5.4. Assume that $p \in(0,1]$ and that $Y$ belongs to $\operatorname{LSub}\left(L^{2}, p\right)$. Assume that the degree $\gamma$ of the drift operator in equation (5.1) satisfies the assumption (5.8). Then, for each s satisfying the condition (5.7) (and the set of such numbers $s$ is nonempty), and for all $q \in(1, \infty), r>0$ such that $r+s<\gamma$,

(i) The process $Y$ has an $U=H^{-s, q}$-valued modification which is a Lévy process.

(ii) For all $t \in[0, T]$, with probability 1 , the stochastic convolution $\int_{0}^{t} e^{(t-s) A_{q}^{\gamma / 2}} d Y(s)$ takes values in $H_{0}^{r, q}(\mathcal{O})$.

Moreover, if $\delta \in\left[0, \gamma-\frac{d}{2}\right)$, then appropriate $r$ and $s$ can be found such that

(iii) For all $t \in[0, T]$, with probability 1 , the stochastic convolution $\int_{0}^{t} e^{(t-s) A_{q}^{\gamma / 2}} d Y(s)$ takes values in $C_{0}^{\delta}(\mathcal{O})$.

Proof. Part (i) follows from Theorem 2.3 and a well known result for Gaussian, see e.g. [5].

Remark 5.5. (a) In the special case of the subordinator process $Z^{\frac{\alpha}{2}}$ with $\alpha \in(0,2)$, i.e. when $\rho$ is defined by formula (2.1) with $\beta=\frac{\alpha}{2}$, the condition (2.4) is satisfied when $\alpha<1$. Below we will show how it is possible to remove the condition $\alpha<1$ by 
applying part (ii) of Theorem 4.1,

(b)

In view of the Remark 5.5] we have the following special case of Theorem 5.4,

Corollary 5.1. Suppose that $\alpha \in(0,1)$, and $\delta \in\left[0, \gamma-\frac{d}{2}\right)$. Then, for an appropriate $q \in(1, \infty)$, for all $t \in[0, T]$, with probability 1 , the stochastic convolution $\int_{0}^{t} e^{(t-s) A_{q}^{\gamma / 2}} d Y^{\alpha}(s)$ takes values in $C_{0}^{\delta}(\mathcal{O})$.

Remark 5.6. It should be obvious that in Corollary 5.1 the number $q$ plays only an auxiliary rôle. Firstly, we define the stochastic integral $\int_{0}^{t} e^{(t-s) A_{q}^{\gamma / 2}} d Y^{\alpha}(s)$ by means of an operator $A_{q}$ which depends $q$. Secondly, we prove that this stochastic integral takes values in the Sobolev space $H_{0}^{r, q}(\mathcal{O})$. Somehow, imprecisely, Corollary 5.1 could be formulated as follows.

Suppose that $\alpha \in(0,1)$ and $\delta \in\left[0, \gamma-\frac{d}{2}\right)$. Then for all $t \in[0, T]$, with probability 1 , the stochastic convolution $\int_{0}^{t} e^{-(t-s)(-\Delta)^{\gamma / 2}} d Y^{\alpha}(s)$ takes values in $C_{0}^{\delta}(\mathcal{O})$.

5.3. OU process driven by a cylindrical $\alpha$-stable processes with $\alpha>1$. The previous argument works for a subordinator process satisfying the condition (2.4) for some $p \in(0,1]$. In this section we consider subordinator processes satisfying the condition (2.4) for some $p \in(1,2)$.

Theorem 5.7. Assume that $p \in(1,2)$ and that $Y$ belongs to $\operatorname{LSub}\left(L^{2}, p\right)$. Assume that the degree $\gamma$ of the drift operator in equation (5.1) satisfies the assumption

$$
\frac{\gamma}{p}>\frac{d}{2}
$$

Then, for each s satisfying the condition (5.7) 1 and for all $q \in[p, \infty), r>0$ such that $r+s<\frac{\gamma}{p}$,

(i) The process $Y$ has a $U=H^{-s, q}$-valued modification which is a Lévy process.

(ii) For all $t \in[0, T]$, with probability 1 , the stochastic convolution $\int_{0}^{t} e^{(t-s) A_{q}^{\gamma / 2}} d Y(s)$ takes values in $H_{0}^{r, q}(\mathcal{O})$.

Moreover, if

$$
\delta \in\left[0, \frac{\gamma}{p}-\frac{d}{2}\right)
$$

then appropriate $r$ and $s$ can be found so that

(iii) For all $t \in[0, T]$, with probability 1 , the stochastic convolution $\int_{0}^{t} e^{(t-s) A_{q}^{\gamma / 2}} d Y(s)$ takes values in $C_{0}^{\delta}(\mathcal{O})$.

\footnotetext{
${ }^{1}$ Note and the set of such numbers $s$ is non-empty.
} 
Proof of Theorem 5.7. We only need to proof the part (iii). Trivially we can find $q \in[p, \infty)$ such that $\delta+\frac{d}{q}+\frac{d}{2}<\frac{\gamma}{p}$. In view of inequality (5.10) in order to show that the condition (4.4) is satisfied, we need to show that we can find $r, s$ such that the conditions (5.11)-(5.7) hold true and $p \frac{r+s}{\gamma}<1$. Since the last condition is equivalent to $r+s<\frac{\gamma}{p}$, the proof is concluded by observing that with $\varepsilon=\frac{1}{3}\left(\frac{\gamma}{p}-\delta-\frac{d}{q}-\frac{d}{2}\right)>0$, it is enough to put $r=\delta+\frac{d}{q}+\varepsilon$ and $s=\frac{d}{2}+\varepsilon$.

Remark 5.8. As in Remark 5.3 the Hilbert space $H=L^{2}$ can be replaced by $H^{\vartheta, 2}(\mathcal{O})$ with, for concreteness $\vartheta \in\left(-\frac{1}{2}, \frac{1}{2}\right)$. The appropriate version of 5.7 can be formulated as follows.

Let $H=H^{\vartheta, 2}(\mathcal{O})$ with $\vartheta \in\left(-\frac{1}{2}, \frac{1}{2}\right)$ and $U=H^{-s, q}(\mathcal{O})$. Assume that $p \in(1,2)$ and that a cylindrical Lévy process $Y$ belongs to the class $\operatorname{LSub}\left(L^{2}, p\right)$, i.e. $Y$ is defined by formula (2.7), where $W$ is a cylindrical Wiener process on the Hilbert space $H=H^{\vartheta, 2}(\mathcal{O})$ with $\vartheta \in\left(-\frac{1}{2}, \frac{1}{2}\right)$, and $Z$ is a subordinator process belonging to the class $\operatorname{Sub}(p)$. Assume that the degree $\gamma$ of the drift operator in equation (5.1) satisfies the assumption

$$
\frac{\gamma}{p}>\frac{d}{2}-\vartheta
$$

Then, for each s satisfying the condition (5.13) and for all $q \in[p, \infty), r>0$ such that $r+s<\frac{\gamma}{p}$,

(i) The process $Y$ has a $U=H^{-s, q}$-valued modification which is a Lévy process.

(ii) For all $t \in[0, T]$, with probability 1 , the stochastic convolution $\int_{0}^{t} e^{(t-s) A_{q}^{\gamma / 2}} d Y(s)$ takes values in $H_{0}^{r, q}(\mathcal{O})$.

Let us also note that the supremum of all numbers $r$ satisfying the conditions (5.13) and (5.17) is equal to $\frac{\gamma}{p}+\vartheta-\frac{d}{2}$.

Corollary 5.2. Suppose that $\alpha \in(0,2)$ and $\delta \in\left[0, \frac{\gamma}{\alpha}-\frac{d}{2}\right)$. Then there exists $q \in(\alpha \vee 1, \infty)$ such that $\delta+\frac{d}{q}+\frac{d}{2}<\frac{\gamma}{\alpha}$ and for all $t \in[0, T]$, with probability 1 , the stochastic convolution $\int_{0}^{t} e^{(t-s) A_{q}^{\gamma / 2}} d Y^{\alpha}(s)$ takes values in $C_{0}^{\delta}(\mathcal{O})$.

Proof of Corollary 5.2. We take $\alpha \in(0,2)$ and put $\beta:=\frac{\alpha}{2}$. Since the subordinator $Z^{\frac{\alpha}{2}}$ satisfies the condition (4.4) with any $p>\alpha$ we can choose $p>\alpha$ such that the condition (5.16) is satisfied. Then the result follows from Theorem 5.7.

Remark 5.9. We infer from the above theorem that as $\alpha \in(0,2)$ decreases, the spatial regularity of the stochastic convolution $\int_{0}^{t} e^{(t-s) A_{q}^{\gamma / 2}} d Y^{\alpha}(s)$ increases.

Remark 5.10. It should be obvious that in Corollary 5.2 the number $q$ plays only an auxiliary rôle. Firstly, we define the stochastic integral $\int_{0}^{t} e^{(t-s) A_{q}^{\gamma / 2}} d Y^{\alpha}(s)$ by means 
of an operator $A_{q}$ which depends $q$. Secondly, we prove that this stochastic integral takes values in the Sobolev space $H_{0}^{r, q}(\mathcal{O})$. Somehow, imprecisely, Corollary 5.2 could be formulated as follows.

Suppose that $\alpha \in(0,2)$ and $\delta \in\left[0, \frac{2}{\alpha}-\frac{d}{2}\right)$. Then for all $t \in[0, T]$, with probability 1 , the stochastic convolution $\int_{0}^{t} e^{(t-s) \Delta} d Y^{\alpha}(s)$ takes values in $C_{0}^{\delta}$.

5.4. Comparisons with Lescot-Röckner [31]. The case of equation (1.6) with $d=1$ and $Y$ such that

$$
\mathbb{E}\left(e^{i\langle Y(t), \phi\rangle}\right)=e^{-t \lambda(\phi)}, \lambda(\phi)=\int_{0}^{1} \phi(\sigma)^{2} d \sigma+\left[\int_{0}^{1} \phi(\sigma)^{2} d \sigma\right]^{\frac{\alpha}{2}}, \phi \in L^{2}(0,1),
$$

for some $\alpha \in(0,2)$, has been studied in [31]. Note that by Theorem [2.3, part $i)$,

$$
\mathbb{E}\left(e^{i\langle X(t), \phi\rangle}\right)=e^{-N_{1}(\phi)-N_{2}(\phi)},
$$

where,

$$
N_{1}(\phi)=\int_{0}^{t} \int_{0}^{1} \mid\left(\left.S(s) \phi(\sigma)\right|^{2} d \sigma d s, N_{2}(\phi)=\int_{0}^{t}\left[\int_{0}^{1} \mid\left(\left.S(s) \phi(\sigma)\right|^{2} d \sigma\right]^{\alpha / 2} d s,\right.\right.
$$

and $S$ is the heat semigroup on $L^{2}(0,1)$.

The paper [31] relies on using the Sazonov topology for the functionals $N_{1}$ and $N_{2}$ and proves that there exist two Hilbert-Schmidt operators $U_{1}$ and $U_{2}$ on the space $H=L^{2}(0,1)$ such that

$$
N_{1}(\phi)=\left\|U_{1} \phi\right\|, N_{2}(\phi) \leq\left\|U_{2} \phi\right\|^{\alpha}, \phi \in H .
$$

The above implies that the functionals $N_{1}$ and $N_{2}$ are continuous in the Sazonov topology so they are generating probability measures on $H$. Hence the authors conclude that the mild solution $X$ takes values in the space $L^{2}(0,1)$. We cover more general case, i.e. we allow $d$ to be $\geq 1$ and consider also fractional Laplacian. Our results in conjunction with the purely Gaussian case as studied for example in [5] imply that also in the mixed case, the solution $X$ of equation (1.5) takes values in the space $C_{0}^{\delta}(0,1)$, for $\delta<\frac{\gamma}{2}-\frac{1}{2}$.

5.5. Diagonal case. We begin with a definition. If $b=\left(b_{j}\right)_{j \in \mathbb{N}}$ is a sequence of positive numbers and $q \in[1, \infty)$, then by $l_{b}^{q}$ we mean a Banach space of all sequences $x=\left(x_{j}\right)_{j=1}^{\infty}$ such that $\left(\sum_{j} b_{j}^{q}\left|x_{j}\right|^{q}\right)^{1 / q}$ is finite. If $a=\left(a_{j}\right)_{j \in \mathbb{N}}$ is a sequence of positive numbers then by $a^{-1}$ we will understand a sequence $a^{-1}:=\left(a_{j}^{-1}\right)_{j \in \mathbb{N}}$.

Let us take two increasing positive sequences $a=\left(a_{j}\right)_{j \in \mathbb{N}}, b=\left(b_{j}\right)_{j \in \mathbb{N}}$ convergent to $\infty$ and two numbers $r, q \in[1, \infty)$. Put

$$
H=l^{2}, U=l_{a^{-1}}^{r}, E=l_{b}^{q} .
$$


It follows from Theorem 2.3 in [12], see also [28], that the embedding $H \hookrightarrow U$ is $\gamma$-radonifying iff

$$
\sum_{j} a_{j}^{-r}<\infty
$$

Let $\lambda=\left(\lambda_{j}\right)_{j \in \mathbb{N}}$ be an increasing positive sequence convergent to $\infty$ and let $\left(e_{j}\right)_{j}$ be the standard ONB of $H$. Define a linear operator $A$ in $H$ by $D(A)=\{h \in H$ : $\left.\sum_{j} \lambda_{j}^{2} h_{j}^{2}<\infty\right\}, A h=\sum_{j} \lambda_{j} h_{j} e_{j}$. Then $A$ is a self-adjoint linear operator in $H$. Let $S(t)$ be the $C_{0}$ semigroup generated by the operator $-A$. Easy calculations show that $S(t)$ has a unique extension to a bounded linear map from $U$ to $E$ and

$$
|S(t)|_{L(U, E)}=\sup _{n} e^{-\lambda_{n} t} b_{n} a_{n}^{r / q} .
$$

Consider now a special case, when for some $\alpha>0, \beta \geq 0$,

$$
a_{n}=\lambda_{n}^{\alpha}, b_{n}=\lambda_{n}^{\beta} \text {. }
$$

Since $\sum_{j} a_{j}^{-r}=\sum_{j} \lambda_{j}^{-r \alpha}$ the embedding $H \hookrightarrow U$ is $\gamma$-radonifying iff

$$
\sum_{j} \lambda_{j}^{-r \alpha}<\infty
$$

We also have

$$
\begin{aligned}
|S(t)|_{L(U, E)} & =\sup _{n}\left[e^{-\lambda_{n} t} \lambda_{n}^{\beta+\frac{r}{q} \alpha}\right] \\
& =\sup _{n}\left[e^{-\lambda_{n} t}\left(t \lambda_{n}\right)^{\beta+\frac{r}{q} \alpha}\right] t^{-\beta-\frac{r}{q} \alpha} \leq c^{*} t^{-\beta-\frac{r}{q} \alpha},
\end{aligned}
$$

where $c^{*}:=\sup _{x \geq 0}\left[e^{-x}(x)^{\beta+\frac{r}{q} \alpha}\right]=e^{-\beta-\frac{r}{q} \alpha}\left(\beta+\frac{r}{q} \alpha\right)^{\beta+\frac{r}{q} \alpha}$.

Thus we infer that if $\left(\beta+\frac{r}{q} \alpha\right) p<1$, then

$$
\int_{0}^{1}|S(t)|_{L(U, E)}^{p} d t<\infty
$$

Hence we infer that for all choices of $\alpha$ and $\beta$ we can find $p \in(0,1)$ such that the condition (5.21) holds.

Consequently, imposing some integrability conditions on the intensity of jumps of the subordinator process $Z$, i.e. $\int_{0}^{1} s^{p / 2} \rho(d s)<\infty$, one can show that the trajectories of the corresponding OU process belong to the space $E$. These considerations can be summarized in the following result.

Theorem 5.11. Suppose that $p \in(0,2]$ and $Z$ is a subordinator process from the class $\operatorname{Sub}(p)$. Suppose that $\lambda=\left(\lambda_{j}\right)_{j \in \mathbb{N}}$ is an increasing positive sequence convergent to $\infty$ and $\alpha, \beta \in \mathbb{R}$ such that $\left(\beta+\frac{r}{q} \alpha\right) p<1$ and $\sum_{j} \lambda_{j}^{-r \alpha}<\infty$. Put $a_{n}=\lambda_{n}^{\alpha}, b_{n}=\lambda_{n}^{\beta}$ and define the spaces $H, E, U$ by formula (5.18), where in the case $p>1$ we assume 
additionally that $q \geq p$. Let $W$ be a $U$-valued, $H$-cylindrical Wiener process and let $Y$ be a Lévy process defined by $Y(t)=W(Z(t)), t \geq 0$. Then for all $t>0$, the stochastic convolution takes values in the space $E$.

Remark 5.12. The additional assumption that $q \geq p$ in the case $p>1$ is made so that the space $E=l_{(b)}^{q}$ is of type $p$.

In a subcase when $r=q$ and $A_{q}$ denotes a linear operator in $X=l^{q}$ defined by $D\left(A_{q}\right)=\left\{h \in l^{q}: \sum_{j} \lambda_{j}^{q} h_{j}^{q}<\infty\right\}, A h=\sum_{j} \lambda_{j} h_{j} e_{j}$, the space $E$ defined above equals to $D\left(A_{q}^{\beta}\right)$. Thus we have the following corollary.

Corollary 5.3. Suppose that $Z$ is a subordinator process such that for some $p \in(0,2]$, $\int_{0}^{1} s^{p / 2} \rho(d s)<\infty$ and $q \in(p \vee 1, \infty)$. Suppose that $\lambda=\left(\lambda_{j}\right)_{j \in \mathbb{N}}$ be an increasing positive sequence convergent to $\infty$ and $\alpha, \beta \in \mathbb{R}$ such that $(\beta+\alpha) p<1$ and $\sum_{j} \lambda_{j}^{-q \alpha}<$ $\infty$. Let $H=l^{2}$ and $X=l^{q}$. Let $W$ be a $D\left(A_{q}^{-\beta}\right)$-valued, $H$-cylindrical Wiener process and define a Lévy process $Y$ by $Y(t)=W(Z(t)), t \geq 0$. Then for all $t>0$, with probability 1 , the stochastic convolution takes values in the space $D\left(A_{q}^{\beta}\right)$.

Remark 5.13. In all these considerations, the space $U$ plays only an auxiliary rôle.

Example 5.1. In the very special case when $\lambda_{n}=n^{2 / d}$ for some $d>0$, for each $\beta \in\left(0, \frac{1}{p}\right)$ we can find $q$ and $\alpha>\frac{d}{2 q}$ such that $\beta<\frac{1}{p}-\alpha$. In that case, for all $t>0$, with probability 1 , the stochastic convolution takes values in the space $D\left(A_{q}^{\beta}\right)$.

We apply now the above method to the equation (1.5)

$$
\left\{\begin{aligned}
d X(t) & =-(-\Delta)^{\gamma} X(t)+d Y(t), t \geq 0 \\
X(0) & =0
\end{aligned}\right.
$$

were $\gamma$ is a positive constant, $H=L^{2}(\mathcal{O})$ with $\mathcal{O}=[0, \pi]^{d}$ and $\Delta$ is the Laplace operator with zero Dirichlet boundary conditions. The Laplace operator and thus also $(-\Delta)^{\gamma}$ are of diagonal type, with respect to eigenfunctions

$$
e_{j}\left(\xi_{1}, \ldots, \xi_{d}\right)=\left(\sqrt{\frac{2}{\pi}}\right)^{d} \sin \left(n_{1} \xi_{1}\right) \ldots \sin \left(n_{d} \xi_{d}\right)
$$

where $j=\left(n_{1}, \ldots, n_{d}\right)$ is a multi-index of natural numbers. The corresponding eigenvalues of the operator $-(-\Delta)^{\gamma}$ are

$$
\lambda_{j}=\left(n_{1}^{2}+\ldots+n_{d}^{2}\right)^{\gamma} .
$$

We set $\beta=0, q=1$ and $b_{j}=1, j \in \mathbb{N}$. Let $E$ be the space of all uniformly and absolutely convergent series

$$
\sum_{j} e_{j}(\xi) x_{j}, \xi \in, ; x=\left(x_{j}\right) \in l_{(b)}^{1} .
$$


Then $E$ can be identified with $l_{(b)}^{1}$ and $E \subset C_{0}\left([0, \pi]^{d}\right)$ with the continuous embedding. Applying now Theorem 5.11, with $a_{j}=\left(\lambda_{j}\right)^{\alpha}, j \in \mathbb{N}$, we arrive at the following result.

Theorem 5.14. Suppose that $p \in\left(0, \frac{2 \gamma}{d} \wedge 1\right)$ and $Z$ is a subordinator process from the class $\operatorname{Sub}(p)$, then the solution of the equation (1.5) takes values in the space $C_{0}\left([0, \pi]^{d}\right)$.

Proof. It is enough to find $\alpha$ and $r$ such that the assumptions of Theorem 5.11 are satisfied. Firstly, we observe that he assumption that $\sum_{j} \lambda_{j}^{-r \alpha}<\infty$ is satisfied iff $r \alpha \frac{2 \gamma}{d}>1$, i.e. $r \alpha<\frac{d}{2 \gamma}$. Secondly, we observe that he assumption that $1>\left(\beta+\frac{r}{q}\right) p$ is satisfied iff $r \alpha<\frac{1}{p}$. Since we assume that $\frac{1}{p}<\frac{d}{2 \gamma}$, the desired numbers $r$ and $\alpha$ can be found and hence we get the result.

Remark 5.15. Actually, the proof above would work also for $p \in\left(1, \frac{2 \gamma}{d} \wedge 2\right)$ provided the space $E$ were of type $p$. We wonder if one could modify this proof so it also works for that range of $p$. However, we do not expect that such a proof would lead to a stronger result that Theorem 5.7 .

\section{Limits TO SPATIAL REGULARITY}

It was shown in the preceding sections that the Ornstein-Uhlenbeck processes evolve in much smaller spaces than the noise process. It seems that the reason for that phenomenon is the analyticity of the semigroup $S(t), t \geq 0$. In this section we will show that, in general, the Ornstein-Uhlenbeck processes do not evolve even in the spaces in which the noise lives. An example of this sort, with the Wiener driving process, was constructed by Dettweiler and van Neerven [18]. These authours have cleverly adapted an example from a joint paper of the current authours with Peszat [13] and showed that there exists a Banach space $E=U$, a $C_{0} \operatorname{semigroup~on~} E$ and an element $f \in E \backslash\{0\}$ such that the solution to the Langevin equation (5.2), i.e.

$$
\left\{\begin{aligned}
d X(t) & =A X(t)+f d W(t), t \geq 0 \\
X(0) & =0
\end{aligned}\right.
$$

where $W$ is standard $\mathbb{R}$-valued Wiener process, does not take values in the space $E$.

We shall show below that a generalization of the results from [13] published recently in [29] leads to a result analogous to the one from [18]. Namely, we will indicate a Banach space $E$, a $C_{0}$ group (with the generator denoted by $A$ ) of linear bounded maps on $E$ and a one-dimensional Lévy process $Y$ such that for some $f \in E$ the mild solution to problem

$$
\left\{\begin{aligned}
d X(t) & =A X(t)+f d Y(t), t \geq 0 \\
X(0) & =0
\end{aligned}\right.
$$


does not take values in $E$. We should point out here that our approach to the wellposedness is different to the one used in [18] and it could be used to give another proof of the main result from [18].

Let $\mathbb{S}=\mathbb{R} / \bmod 2 \pi$ be the unit circle and $E=C(\mathbb{S})$ the Banach space of all continuous real-valued functions on $\mathbb{S}$. Note that $E$ can be identified, and this identification will be used below, with the space $\tilde{E}$ of all continuous $2 \pi$-periodic functions from $\mathbb{R} \rightarrow \mathbb{R}$. Given $f \in E$ we will denote by $\tilde{f}$ the corresponding element of $\tilde{E}$. Let $A=d / d \theta$ the infinitesimal generator of the rotation group $S=\{S(t)\}_{t \in \mathbb{R}}$ on $E$, defined via the above identification by $\tilde{S}_{t}(\tilde{f})=\tilde{f}(\cdot+t)$.

Theorem 6.1. Assume that the real valued Lévy process $Y$ has trajectories with unbounded variation. There exists $f \in E$ such that a.s. the mild solution to problem (6.2) does not take values in E.

Proof. It follows from the proof of Theorem 3.1, p. 599 in [29], that we can find $f \in E$ such that the process

$$
\int_{0}^{2 \pi} \tilde{f}(z-s) d Y(s), \quad z \in[0,2 \pi],
$$

has unbounded paths almost surely. On the other hand, since $\tilde{f}$ is $2 \pi$-periodic, the solution $X$ of the process (6.2) satisfies

$$
\begin{aligned}
X(2 \pi) & =\int_{0}^{2 \pi} S(2 \pi-s) f d Y(s)=\int_{0}^{2 \pi} \tilde{f}(\cdot+2 \pi-s) d Y(s) \\
& =\int_{0}^{2 \pi} \tilde{f}(\cdot-s) d Y(s) .
\end{aligned}
$$

Hence,

$$
X(2 \pi, z)=\int_{0}^{2 \pi} \tilde{f}(z-s) d Y(s), \text { for a.a. } z \in[0,2 \pi]
$$

In this way the proof in complete.

Remark 6.2. The proof of Theorem 6.1 was based on showing that there exists $f \in E$ such that $X(2 \pi) \notin E$ a.s. In this way, our result is a direct consequence of [29]. We believe that as in [13] one can show that set of all $f \in E$ such that $X(2 \pi) \notin E$ a.s. is of 2nd category in $E$.

Note that Remark 4.5(iv) is applicable here but with a different choice of space $E$. We get the following result which, as it should be noted, does not contradict Theorem 6.1. 
Theorem 6.3. Assume that $Z \in \operatorname{Sub}(p)$ with $p \in(1,2]$. Let $E=W^{\theta, q}(\mathbb{S})$ with $q \in[p, \infty)$ and $\theta \geq 0$. Then, for each $f \in W^{\theta, q}(\mathbb{S})$, there exists a mild solution $X$ to the problem (6.2) and $X$ is an $W^{\theta, q}(\mathbb{S})$-valued cádlág process.

In particular, if $\theta>\frac{1}{q}, f \in W^{\theta, q}(\mathbb{S})$, the mild solution $X$ to the problem (6.2) is a $C(\mathbb{S})$-valued cádlág process.

Proof. The first part of the Theorem is a consequence of Remark 4.2(iv) since the Besov-Slobodetskii space $W^{\theta, q}(\mathbb{S})$ is a Banach space of type $p$. The second part is a consequence of the first one and the the Sobolev embedding Theorem in view of which, under the assumptions of the Theorem, $W^{\theta, q}(\mathbb{S}) \hookrightarrow C(\mathbb{S})$.

\section{Time IRREGULARITY}

We pass now to the time regularity and show that in general trajectories are not even locally bounded. We will work in the framework of section 4. In particular, we consider a triple of spaces $E, H$ and $U$ such that the middle one is a Hilbert space and the other two are Banach spaces and (4.1) hold.

We consider an $U$-valued Wiener process $W=(W(t))_{t \geq 0}$ (with RKHS $H$ ), a subordinator process $Z=(Z(t))_{t \geq 0}$ and an $U$-valued Lévy proves $Y=(Y(t))_{t \geq 0}$ by the subordination formula (1.2), i.e.

$$
Y(t):=W(Z(t)), t \geq 0 .
$$

Now we assume that $-A$ is a generator of an analytic $\mathrm{S}=\left(e^{t A}\right)_{t \geq 0}$ semigroup in $E$ and $U$ and consider the stochastic convolution process

$$
X(t)=\int_{0}^{t} e^{(t-s) A} d Y(s), t \geq 0 .
$$

For simplicity, we only consider the case $p \in(1,2]$. As in section 4 we decompose the process $Y$ into two independent parts, see equalities (4.8)-(4.9), both being cádlág , and consider the processes $X_{1}$ and $X_{2}$ defined by

$$
\begin{aligned}
& X_{1}(t)=\int_{0}^{t} e^{(t-s) A} d Y_{1}(s), t \geq 0, \\
& X_{2}(t)=\int_{0}^{t} e^{(t-s) A} d Y_{2}(s), t \geq 0 .
\end{aligned}
$$

Our first aim is to prove the following generalization of [40, Theorem 9.2.4, p.269].

Theorem 7.1. In the framework of Theorem 4.4, if $F$ is a separable Banach space such that

(i) $E \subset F \subset U$ and $\mu_{1}(F)=0$, i.e. $H$ is not embedded into $F$ in a $\gamma$-radonifying way, 
(ii) $e^{t A}$ is a bounded linear map from $U$ to $F$ for $t>0$ and

$$
\left\{x \in U: \sup _{t \in(0,1]}\left|e^{t A} x\right|_{F}<\infty\right\} \subset F .
$$

Then with positive probability, the process $\int_{0}^{t} e^{(t-s) A} d Y(s), t \geq 0$, is not $F$-valued cádlág.

In particular, if a Banach space $F$ is such that $F \subset H$ and condition (ii) holds, then with positive probability, the process $\int_{0}^{t} S(t-r)(r) d Y(r), t \geq 0$, is not $F$-valued cádlág .

Remark 7.2.(i) In view of Theorem 4.1, the first part of condition (i) in Theorem 7.1 implies that $X$ is an $F$-valued process.

(ii) If $-A$ is an infinitesimal generator of an analytic and compact semigroup $\mathrm{S}=$ $\left(e^{t A}\right)_{t \geq 0}$ in $U$ and $F=D\left(A^{\beta}\right)$, for $\beta>0$, with the graph norm, then the condition (ii) in Theorem 7.1 is satisfied. Indeed, $A^{\beta}$ is a closed operator.

Proof. Let us fix $T>0$. Let $0<\tau_{1}<\tau_{2}<\tau_{3}<\cdots$ be the jump times of the process $Y_{2}$ and $\Delta Y_{2}\left(\tau_{k}\right)=\Delta Y\left(\tau_{k}\right)-Y\left(\tau_{k}-\right)$, for all $k$. Since $X_{2}(t)=0$ for $t \in\left[0, \tau_{1}\right)$ and $\tau_{1}$ is an exponential r.v. with mean value $>0$, we infer that $q_{2}:=$ $\mathbb{P}\left(X_{2}\right.$ is bounded on $\left.[0, T]\right)$ is strictly positive. Assume for the time being that we have shown that also $q_{2}<1$, i.e.

$$
1-q_{2}:=\mathbb{P}\left(X_{2} \text { is unbounded on }[0, T]\right) \text { is strictly positive. }
$$

Denote by $q_{1}$ the probability that $X_{1}$ is is bounded on $[0, T]$. Note finally that since $Y_{1}$ and $Y_{2}$ are independent processes so are $X_{1}$ and $X_{2}$. We have then the following simple chain of inequalities.

$$
\begin{aligned}
& \mathbb{P}(X \text { is ubnd on }[0, T]) \geq \mathbb{P}\left(X_{1} \text { is bnd and } X_{2} \text { is ubnd on }[0, T]\right. \\
\text { or } \left.X_{1} \text { is ubnd and } X_{2} \text { is bnd on }[0, T]\right) & \mathbb{P}\left(X_{1} \text { is bnd and } X_{2} \text { is ubnd on }[0, T]\right) \\
& +\mathbb{P}\left(X_{1} \text { is ubnd and } X_{2} \text { is bnd on }[0, T]\right) \\
= & \mathbb{P}\left(X_{1} \text { is bnd on }[0, T]\right) \mathbb{P}\left(X_{2} \text { is ubnd on }[0, T]\right) \\
& +\mathbb{P}\left(X_{1} \text { is ubnd on }[0, T]\right) \mathbb{P}\left(X_{2} \text { is bnd on }[0, T]\right) \\
= & q_{1}\left(1-q_{2}\right)+\left(1-q_{1}\right) q_{2} .
\end{aligned}
$$

Note that the number $q_{1}\left(1-q_{2}\right)+\left(1-q_{1}\right) q_{2}$ is a convex combination of numbers $q_{2}$ and $1-q_{2}$ and since both of them are strictly positive, so is $q_{1}\left(1-q_{2}\right)+\left(1-q_{1}\right) q_{2}$. This proves that $\mathbb{P}(X$ is ubnd on $[0, T])>0$ and hence the proof is complete. We 
only need to prove claim (7.4). For this aim let us notice that for $\omega \in \Omega$ and $t \in\left(\tau_{1}(\omega), \tau_{2}(\omega)\right)$,

$$
X_{2}(t, \omega)=e^{\left(t-\tau_{1}(\omega)\right) A} \Delta Y\left(\tau_{1}(\omega)\right)=e^{\left(t-\tau_{1}(\omega)\right) A}\left(W\left(\beta \tau_{1}(\omega), \omega\right)-W\left(\beta \tau_{1}(\omega), \omega\right)\right) .
$$

Since with positive probability $\left(W\left(\beta \tau_{1}(\omega), \omega\right)-W\left(\beta \tau_{1}(\omega), \omega\right)\right) \notin F$, by (ii), we infer that $\lim \sup _{t \searrow \tau_{1}}\left|X_{2}(t)\right|_{F}=\infty$.

\section{Applications to nOnlinear EQUations}

Let us consider, for a moment, nonlinear equation on a Banach space $E$,

$$
d u(t)=(A u(t)+F(u(t)) d t+d Y(t), t \geq 0, u(0)=x,
$$

which can be rewritten as an integral equation

$$
u(t)=S(t) x+\int_{0}^{t} S(t-s) F\left(\left(u(s)+Y_{A}(s)\right) d s, t \geq 0 .\right.
$$

where $Y_{A}$ is an Ornstein- Uhlenbeck process and thus solution to the equation with $F=0$. As we already know, in general, $Y_{A}$ has locally unbounded trajectories and this makes the equation 8.2 rather singular. However, if for instance $F$ is Lipschitz on $E$ then for the solvability of $(\underline{8.2})$ is enough that trajectories of $Y_{A}$ are in some $L^{p}([0, T], E)$.

8.1. Time integrability of OU trajectories. It turns out that in trajectories of the process $X$ have important integrability properties. Taking into account the decomposition (4.5) we write,

$$
X(t)=\int_{0}^{t} S(t-s) d Y_{1}(s)+\int_{0}^{t} S(t-s) d Y_{2}(s)=X_{1}(t)+X_{2}(t),
$$

and from inequalities (4.14) and (4.17) we have the following result.

Theorem 8.1. If $p \in(1,2], Z$ is an subordinator process from the class $\operatorname{Sub}(p), E$ is a separable type $p$ Banach space, then for some constant $C>0$,

$$
\mathbb{E} \int_{0}^{T}\left|X_{1}(t)\right|_{E}^{p} d t \leq C T \int_{0}^{T}\left|e^{r A}\right|_{\mathcal{L}(U, E)}^{p} d r
$$

Hence, if $\int_{0}^{T}\left|e^{r A}\right|_{\mathcal{L}(U, E)}^{p} d r<\infty$, then $\mathbb{E} \int_{0}^{T}\left|X_{1}(t)\right|_{E}^{p} d t<\infty$ as well.

In particular, if for some $C>0$ and some $\theta \in\left(0, \frac{1}{p}\right)$,

$$
\left|e^{r A}\right|_{\mathcal{L}(U, E)} \leq C r^{-\theta}, r>0,
$$

then, for some $C>0$ and all $T>0$,

$$
\mathbb{E} \int_{0}^{T}\left|X_{1}(t)\right|_{E}^{p} d t \leq C T^{2-\theta p}
$$


Proof. By the Fubini Theorem we have the following chain of inequalities.

$$
\begin{aligned}
\mathbb{E} \int_{0}^{T}\left|X_{1}(t)\right|_{E}^{p} d t & \leq C \int_{0}^{T} \int_{0}^{t}\left|e^{(t-s) A}\right|_{\mathcal{L}(U, E)}^{p} d s d t \\
=C \int_{0}^{T} \int_{0}^{t}\left|e^{r A}\right|_{\mathcal{L}(U, E)}^{p} d r d t & \leq \int_{0}^{T} \int_{0}^{T}\left|e^{r A}\right|_{\mathcal{L}(U, E)}^{p} d r d t \\
& =C T \int_{0}^{T}\left|e^{r A}\right|_{\mathcal{L}(U, E)}^{p} d r .
\end{aligned}
$$

This proves inequality (8.3) while the proof of (8.5) follows the same lines.

Remark 8.2. Note that assumption (8.4) is sufficient for both the existence of $E$ valued modification of $X$ and for the $p$-integrability of the $E$-norm of trajectories of the process $X$.

Corollary 8.1. Under the assumptions of Theorem 8.1, with probability 1, for all $T>0$,

$$
\int_{0}^{T}|X(t)|_{E}^{p} d t<\infty
$$

Proof. It is enough to consider the case of the process $X_{2}$. Then, compare with (4.11), we have

$$
X_{2}(t)=\int_{0}^{t} e^{(t-s) A} d Y_{2}(s):=\sum_{\tau_{k} \leq t} e^{\left(t-\tau_{k}\right) A} \Delta Y\left(\tau_{k}\right), t \geq 0,
$$

where $0<\tau_{1}<\tau_{2}<\tau_{3}<\cdots$ are the jump times of $Y_{2}$ and $\Delta Y_{2}\left(\tau_{k}\right)=\Delta Y\left(\tau_{k}\right)-$ $Y\left(\tau_{k}-\right)$, for all $k$. Note that, c.f. with the proof of Theorem 7.1 , that the process $X_{2}$ is continuous as a $U$-valued but as an E-valued process, $X_{2}$ has almost surely, unbounded trajectories.

For each $k$ and arbitrary $\omega \in \Omega$,

$$
X_{2}(t)=S\left(t-\tau_{k}\right)\left[X_{2}\left(\tau_{k}-\right)+\Delta Y_{2}\left(\tau_{k}\right)\right], t \in\left[\tau_{k}, \tau_{k+1}\right) .
$$

Note that $X_{2}(t)=0$ for $t \in\left[0, \tau_{1}\right)$ so that $\int_{0}^{\tau_{1}}\left|X_{2}(t)\right|_{E}^{p} d t=0<\infty$. If $k \geq 1$, then we have

$$
\int_{\tau_{k}}^{\tau_{k+1}}\left|X_{2}(t)\right|_{E}^{p} d t \leq C \int_{0}^{\tau_{k+1}-\tau_{k}}\left|e^{r A}\right|_{\mathcal{L}(U, E)}^{p} d r\left|X_{2}\left(\tau_{k}\right)+\Delta Y_{2}\left(\tau_{k}\right)\right|_{U}^{p} .
$$

Since the RHS of inequality (8.7) is $<\infty$ almost surely, the proof is complete.

Remark 8.3. The proof of Corollary 8.1 shows that if $q \in[1, \infty)$ and $\int_{0}^{T}\left|e^{r A}\right|_{\mathcal{L}(U, E)}^{q} d r<$ $\infty$, then with probability 1 , for all $T>0$,

$$
\int_{0}^{T}\left|X_{2}(t)\right|_{E}^{q} d t<\infty
$$


8.2. Burgers equation with additive Lévy noise. The Burgers equation with Lévy type noise has been considered in [50], [40] and recently in [20]. We will test here applicability of the results obtained in previous sections. However our results are not directly comparable with that of [50] because we deal with different form of noise. As we have pointed our earlier the most recent paper [20] considers the case of the compound Poisson noise and in fact deals with deterministic Burgers equation.

As a preparation we consider first integrability of the trajectories of the O-U process generated by the following Langevin equation on a one-dimensional domain $(0,1)$, i.e.

$$
\left\{\begin{aligned}
d X(t) & =\Delta X(t)+d Y(t), t \geq 0 \\
X(0) & =0
\end{aligned}\right.
$$

with Dirichlet boundary conditions, where $Y$ is a Lévy process defined by (9.3) with $\beta \in \operatorname{Sub}(p), p \in(1,2]$ and $W$ being a $H$-cylindrical Wiener process with $H=$ $H^{\theta, 2}(0,1)$ for some $\theta \in\left(0, \frac{1}{2}\right)$. We shall prove the following.

Proposition 8.4. Under the above assumptions, with probability 1 , for all $T>0$,

$$
\int_{0}^{T}|X(t)|_{L^{4}}^{4} d t<\infty .
$$

Proof. By Theorem 5.7, or rather Remark 5.8, and Corollary 8.1, for each $r<\frac{2}{p}-\frac{1}{2}+\vartheta$ and $s>\frac{1}{2}-\vartheta$ such that $r+s<\frac{2}{p}$, and all $q \in[p, \infty)$, the process $X$ is $H^{-s, q}(0,1)$ cádlág and hence is $H^{-s, q}(0,1)$-valued bounded, takes values in $H^{r, q}(0,1)$ and

$$
\int_{0}^{T}|X(t)|_{H^{r, q}}^{p} d t<\infty .
$$

Let us choose a particular $s$, i.e. such that $\frac{1}{2}-\vartheta<s<\frac{1}{2}$. Put then $r=s\left(\frac{4}{p}-1\right)$. Then $r+s=\frac{4 s}{p}<\frac{2}{p}$ and $r<\frac{2}{p}-s<\frac{2}{p}-\frac{1}{2}+\vartheta$. This means that the numbers $r$ and $s$ satisfy the assumptions of Theorem [5.7, Remark [5.8 and Corollary 8.1. Thus

$$
\int_{0}^{T}|X(t)|_{H^{r, q}}^{p} d t<\infty \text { and } \sup |X(t)|_{H^{-s, q}}<\infty \text { a.s. }
$$

On the other hand, y equality (5.4),

$$
L^{q}(0,1)=H^{0, q}(0,1)=\left[H^{-s, q}(0,1), H^{r, q}(0,1)\right]_{\theta},
$$

where $\theta \in(0,1)$ satisfy $\beta=(1-\theta)(-s)+\theta r$, i.e. $\theta=\frac{s}{s+r}$. Hence,

$$
|u|_{L^{q}}=|u|_{H^{-s, q}}^{\frac{r}{r+s}}|u|_{H^{r, q}}^{\frac{s}{r+s}}, \quad u \in H^{r, q}(0,1) .
$$


Hence, by the Hölder inequality, with $q=4$, since $\frac{4 s}{r+s}=p$, we have

$$
\begin{aligned}
\int_{0}^{T}|X(t)|_{L^{4}}^{4} d t & \leq \int_{0}^{T}|X(t)|_{H^{-s, q}}^{\frac{4 r}{r+s}}|X(t)|_{H^{r, q}}^{\frac{4 s}{r+s}} d t \\
& \leq\left(\sup _{t \in[0, T]}|X(t)|_{H^{-s, q}}\right)^{\frac{4 r}{r+s}} \int_{0}^{T}|X(t)|_{H^{r, q}}^{p} d t<\infty \text { a.s. }
\end{aligned}
$$

This concludes the proof.

We pass now to the Burgers equation. We formulate a result which, in view of Proposition 8.4, can be proved following the lines of [9]. Thus let us consider equation

$$
d u+[\mathrm{A} u+B(u)] d t=f d t+d Y(t), \quad t \geq 0
$$

with the initial condition

$$
u(0)=u_{0},
$$

where $B(u)=u u_{x}=\frac{1}{2} \frac{d}{d x}\left(u^{2}\right), u_{0} \in \mathrm{H}=L^{2}(0,1), \mathrm{V}=H_{0}^{1,2}(0,1)$ and $f \in \mathrm{V}^{\prime}=$ $H^{-1,2}(0,1)$ and $Y$ is a Lévy process defined by (9.3) with $\beta \in \operatorname{Sub}(p), p \in(1,2]$ and $W$ being a $H^{\theta, 2}(0,1)$-cylindrical Wiener process for some $\theta \in\left(0, \frac{1}{2}\right)$. Let us notice that $\mathrm{V}$ is a Hilbert space with norm $\|u\|^{2}:=\int_{0}^{1}|\nabla u(x)|^{2} d x$ which is equivalent with the norm inherited from the Sobolev space $H^{1,2}(0,1)$, i.e. $|u|^{2}=\int_{0}^{1}|\nabla u(x)|^{2} d x+$ $\int_{0}^{1}|u(x)|^{2} d x$. The above problem (8.14) is of a similar form as the stochastic NavierStokes Equations, however with one essential difference. The nonlinearity $B$ defined above still satisfies the condition

$$
(B(u), u)=0, u \in \mathrm{V}
$$

but its symmetric bilinear counterpart defined by $B(u, v)=\frac{1}{2} \frac{d}{d x}(u v)$ no longer satisfies the stronger condition $(B(u, v), v)=0$ (even for very smooth functions $u, v$ ).

We propose the following definition of a solution to problem (8.14)-(8.15). For this let us fix a number $s$ such that $\frac{1}{2}-\vartheta<s<\frac{1}{2}$.

Definition 8.5. An H-valued $\left(\mathcal{F}_{t}\right)_{t \geq 0}$ adapted and $H^{-s, 4}(0,1)$-valued cádlág process $u(t), t \geq 0$ is a solution to 8.14)-(8.15) iff

$$
\sup _{0 \leq t \leq T}|u(t)|_{\mathrm{H}}^{2}+\int_{0}^{T}|u(t)|_{L^{4}(0,1)}^{4} d t<\infty \quad \text { for each } T>0 \text { a.s. }
$$

and for any $\psi \in \mathrm{V} \cap H^{2,2}(0,1)$ and $t>0$, the following holds a.s.

$$
\begin{aligned}
(u(t), \psi)-\left(u_{0}, \psi\right) & -\int_{0}^{t}(u(s), \Delta \psi) d s-\frac{1}{2} \int_{0}^{t}\left(u^{2}(s), \nabla \psi\right) d s \\
& =\int_{0}^{t}(f, \nabla \psi) d s+\langle\psi, Y(t)\rangle .
\end{aligned}
$$


Theorem 8.6. For any $u_{0} \in \mathrm{H}$ there exists a unique solution $u(t), t \geq 0$, to the stochastic Burgers Equation (8.14)-(8.15).

Since the construction of the solution given above is pathwise, as a byproduct of it we can show that there exists a Random Dynamical System corresponding to the problem (8.14)-(8.15). It's properties will not be however studied in the current publication. One can consult [9] for the Gaussian case.

A tool for studying the existence and uniqueness of solutions for the problem 8.14 8.15) is the following result whose proof can be found in [9]. Before we formulate it let denote by $\mathcal{H}^{1,2}(0, T)$ the space of all functions $v \in L^{2}\left(0, T ; \mathrm{V} \cap H^{2,2}(0,1)\right.$ such that $v^{\prime} \in L^{2}\left(0, T ; \mathrm{V}^{\prime}\right)$. Let us recall a well known fact, see e.g. [33], that each element $v \in \mathcal{H}^{1,2}(0, T)$ is equal a.e. to a continuous $H$-valued function defined on the closed interval $[0, T]$ and that the well defined embedding $\mathcal{H}^{1,2}(0, T) \hookrightarrow C([0, T] ; H)$ is bounded.

Proposition 8.7. Assume that $z \in L^{4}\left(0, T ; L^{4}(0,1)\right), g \in L^{2}\left(0, T ; \mathrm{V}^{\prime}\right)$ and $v_{0} \in \mathrm{H}$. Then, there exists a unique $v \in \mathcal{H}^{1,2}(0, T)$ such that

$$
\begin{aligned}
\frac{d v}{d t} & +A v+B(v, z)+B(z, v)+B(v, v)=g, \quad t \geq 0, \\
v(0) & =v_{0} .
\end{aligned}
$$

Moreover, with

$$
\begin{aligned}
K^{2} & :=e^{2 \int_{0}^{T}|z(s)|_{L^{4}}^{4} d s}, L^{2}:=\left|v_{0}\right|^{2}+2 \int_{0}^{T}|g(s)|_{\mathrm{V}^{\prime}}^{2} d s, \\
M^{2} & :=\left|v_{0}\right|^{2}+9 K L \int_{0}^{T}|z(t)|_{L^{4}}^{4} d t+\int_{0}^{T}|g(t)|_{\mathrm{V}^{\prime}}^{2} d t \\
N & :=|g|_{L^{2}\left(0, T ; \mathrm{V}^{\prime}\right)}+2 K L M|z|_{L^{4}\left(0, T ; L^{4}(0,1)\right)}^{2}+\frac{T^{1 / 4}}{\sqrt{2}} K^{3 / 2} L^{1 / 2},
\end{aligned}
$$

we have

$$
\begin{aligned}
\sup _{t \in[0, T]}|v(t)|^{2} & \leq K^{2} L^{2}, \int_{0}^{T}|\nabla v(t)|^{2} d t \leq M^{2} \\
\int_{0}^{T}\left|v^{\prime}(t)\right|_{\mathrm{V}^{\prime}}^{2} d t & \leq N^{2}, \int_{0}^{T}|v(t)|_{L^{4}(0,1)}^{4} d t \leq 2 T^{1 / 2} K^{3} L^{3} M .
\end{aligned}
$$

Finally, the map $L^{2}\left(0, T ; \mathrm{V}^{\prime}\right) \times \mathrm{H} \ni\left(g, v_{0}\right) \mapsto v \in \mathcal{H}^{1,2}(0, T)$, where $v$ is the unique solution to 8.17)-(8.18), is real analytic.

Remark 8.8. Open Problem. Does there exists a solution to the stochastic Burgers Equation (8.14)- (8.15) when the Lévy process $Y$ is defined by (9.3) with $\beta \in \operatorname{Sub}(p)$, 
$p \in(1,2]$ and $W$ being a $H$-cylindrical Wiener process with $H=H^{0,2}(0,1)=$ $L^{2}(0,1) ?$

\section{Generalizations to non-autonomous equations}

Without much effort some results can be generalized to the non-autonomous case. - We use some results of Acquistapace and Terreni [3] as presented in [51].

Let us assume that $(A(t), D(A(t)))_{t \in[0, T]}$ is a family of closed and densely defined linear operators on a Banach space $E$.

We say that condition $(A T)$ is satisfied if the following two conditions hold:

(AT1) There exist constants $\lambda_{0} \in \mathbb{R}, K \geq 0$, and $\phi \in\left(\frac{\pi}{2}, \pi\right)$ such that for all $t \in[0, T]$, $\Sigma\left(\phi, \lambda_{0}\right) \subset \varrho(A(t))$, where $\Sigma\left(\phi, \lambda_{0}\right)=\left\{\lambda_{0}\right\} \cup\left\{\lambda \in \mathbb{C} \backslash\left\{\lambda_{0}\right\}:\left|\arg \left(\lambda-\lambda_{0}\right)\right| \leq \phi\right\}$

$$
\|R(\lambda, A(t))\| \leq \frac{K}{1+\left|\lambda-\lambda_{0}\right|}, \text { for all } \lambda \in \Sigma\left(\phi, \lambda_{0}\right) .
$$

(AT2) There exist constants $L \geq 0$ and $\mu, \nu \in(0,1]$ with $\mu+\nu>1$ such that for all $\lambda \in \Sigma(\phi, 0)$ and $s, t \in[0, T]$, with $A_{\lambda_{0}}(t)=A(t)-\lambda_{0}$,

$$
\left\|A_{\lambda_{0}}(t) R\left(\lambda, A_{\lambda_{0}}(t)\right)\left(A_{\lambda_{0}}(t)^{-1}-A_{\lambda_{0}}(s)^{-1}\right)\right\| \leq L|t-s|^{\mu}(|\lambda|+1)^{-\nu} .
$$

Below we will denote $\kappa_{\mu, \nu}=\mu+\nu-1$. Note that by (AT2) $\kappa_{\mu, \nu} \in(0,1]$.

If the assumption (AT1) is satisfied and the domains are constant, i.e. $D(A(0))=$ $D(A(t)), t \in[0, T]$, then the Hölder continuity of a function $[0, T] \ni t \mapsto A(t) \in$ $\mathcal{L}(D(A(0)), E)$ with exponent $\eta$, implies that the condition (AT2) is satisfied with $\mu=$ $\eta$ and $\nu=1$, see [3, Section 7]. The conditions in that case reduce to the conditions in the theory of Sobolevskii and Tanabe for constant domains, cf. [34, 38, 45].

The following result is known, see e.g. [3, Theorems 6.1-6.4] and [52, Theorem $2.1])$.

Theorem 9.1. If condition (AT) holds, then there exists a unique strongly continuous evolution family $(P(t, s))_{0 \leq s \leq t \leq T}$ that solves (9.1) on $D(A(s))$, i.e.

$$
\begin{aligned}
& u^{\prime}(t)=A(t) u(t), \quad t \in[s, T], \\
& u(s)=x .
\end{aligned}
$$

and for all $x \in E, P(t, s) x$ is a classical solution of (9.1). Moreover, $(P(t, s))_{0 \leq s \leq t \leq T}$ is continuous on $0 \leq s<t \leq T$ and there exists a constant $C>0$ such that for all $0 \leq s<t \leq T$ and all $0 \leq \beta \leq \alpha \leq 1$,

$(9.2)\|P(t, s) x\|_{(E, D(A(t)))_{\alpha, 2}} \leq C(t-s)^{\beta-\alpha}\|x\|_{(E, D(A(s)))_{\beta, 2}}, x \in(E, D(A(s)))_{\beta, 2}$, where $(E, D(A(s)))_{\beta, 2}$ denotes the real interpolation space. 
The following result is a generalization of Theorem 4.4 (and of Remark 4.5 (iv)) to a non-autonomous case.

We assume that a triple of spaces $E, H$ and $U$ such that the middle one is a Hilbert space and the other two are separable Banach spaces is such that condition (4.1) is satisfied.

We assume that $W=(W(t))_{t>0}$ is an $H$-cylindrical $U$-valued Wiener process. Assume that $Z=(Z(t))_{t>0}$ is a subordinator process belonging to the class $\operatorname{Sub}(p)$, with $p \in(1,2]$, and, as in Theorem [2.3, $Y=(Y(t))_{t \geq 0}$ is an $U$-valued Lévy process defined by the subordination formula (1.2), i.e.

$$
Y(t):=W(Z(t)), t \geq 0 .
$$

Under the above assumptions we have the following generalization of Theorem 4.1 (ii).

Theorem 9.2. Assume that the conditions (AT1)-(AT2) are satisfied and that the Banach space $E$ is of type $p$, with $p \in(1,2]$. Assume that $T>0, \beta<0<\alpha$ and that $U$ is a separable Banach space such that $U \subset(E, D(A(t)))_{\beta, 2}$, for all $t \in[0, T]$, continuously uniformly in $t$. Assume also that

$$
p(\alpha+\beta)<1 .
$$

Let $X=(X(t))_{t \in[0, T]}$ be the mild solution to the problem

$$
\left\{\begin{aligned}
d X(t) & =A(t) X(t) d t+d Y(t), t \geq 0 \\
X(0) & =0
\end{aligned}\right.
$$

given by formula

$$
X(t)=\int_{0}^{t} P(t, s) d Y(s), t \in[0, T] .
$$

Then, for each $t \in[0, T], X(t)$ takes a.s. values in the space $(E, D(A(t)))_{\alpha, 2}$.

Proof. The proof of this result follows the lines of the proof of Theorem 4.1 (ii) with the formula (9.6) playing the rôle of the integral $\int_{0}^{t} \Psi(s) d Y(s)$ and the assumption (9.4) together with the inequality (9.2) being a replacement of the condition (4.4).

\section{REFERENCES}

[1] D. Applebaum, Lévy processes and stochastic calculus, Cambridge Studies in Advanced Mathematics, 93, Cambridge University Press, Cambridge, 2004.

[2] P. ACQUistapace, Evolution operators and strong solutions of abstract linear parabolic equations, Differential Integral Equations 1 (1988), no. 4, 433-457.

[3] P. Acquistapace and B. Terreni, A unified approach to abstract linear nonautonomous parabolic equations, Rend. Sem. Mat. Univ. Padova 78 (1987), 47-107. 
[4] Z. Brzeźniak, Stochastic partial differential equations in M-type 2 Banach spaces, Potential Anal. 4, no. 1, 1-45 (1995)

[5] Z. Brzeźniak, On stochastic convolution in Banach spaces and applications, Stochastics Stochastics Rep. 61, no. 3-4, 245-295 (1997)

[6] Z. Brzez'niak and L. Debbi, On stochastic Burgers equation driven by a fractional Laplacian and space-time white noise, in STOCHASTIC DIFFERENTIAL EQUATIONS: THEORY AND APPLICAtions, 135-167, a volume in honor of Professor Boris L. Rozovskii, edts.: P. H. Baxendale and S. V. Lototsky, Interdiscip. Math. Sci. 2, World Sci. Publ., Hackensack, NJ, 2007.

[7] Z. Brzeźniak and K. D. Elworthy, Stochastic differential equations on Banach manifolds, Methods Funct. Anal. Topology 6, no. 1, 43-84 (2000)

[8] Z. Brzeźniak, E. Hausenblas, Maximal regularity for stochastic convolutions driven by Lévy processes, submitted

[9] Z. Brzeźniak, Asymptotic compactness and absorbing sets for stochastic Burgers' equations driven by space-time white noise and for some two-dimensional stochastic Navier-Stokes equations on certain unbounded domains, STOCHASTIC PARTIAL DIFFERENTIAL EQUATIONS AND Applications - VII, 35-52, Lect. Notes Pure Appl. Math., 245, Chapman \& Hall/CRC, Boca Raton, FL, 2006.

[10] Z. Brzeźniak and Y. Li, Asymptotic compactness and absorbing sets for $2 D$ Stochastic NavierStokes equations on some unbounded domains, Trans. Amer. Math. Soc. 358, no. 12, 5587-5629 (2006)

[11] Z. Brzeźniak, J. van Neerven, Stochastic convolution in separable Banach spaces and the stochastic linear Cauchy problem, Studia Math. 143, no. 1, 43-74 (2000)

[12] Z. Brzeźniak, J. van Neerven, Space-time regularity for linear stochastic evolution equations driven by spatially homogeneous noise, J. Math. Kyoto Univ. 43, no. 2, 261-303 (2003)

[13] Z. Brzeźniak, S. Peszat and J. Zabczyk, Continuity of stochastic convolutions, Czechoslovak Math. J. 51(126), 679-684 (2001)

[14] C. Chalk, Nonlinear evolutionary equations in Banach spaces with fractional time derivative, PhD thesis; The University of Hull, Kingston upon Hull, UK, 2006.

[15] A. Chojnowska-Michalik, On processes of Ornstein-Uhlenbeck type in Hilbert space, Stochastics 21, no. 3, 251-286 (1987)

[16] G. Da Prato and J. Zabczyk, Stochastic Equations in Infinite Dimensions, CUP 1992.

[17] G. Da Prato and A. Debussche and R. Temam, Stochastic Burgers' equation, NoDEA (Nonlinear Differential Equations Appl.) 1, no. 4, 389-402 (1994).

[18] J. Dettweiler and J. van Neerven, Continuity versus nonexistence for a class of linear stochastic Cauchy problems driven by a Brownian motion, Czechoslovak Math. J. 56(131) (2006), no. 2, $579-586$.

[19] N. Dinculeanu, Vector integration and Stochastic integration in Banach SPACES, Pure and Applied Mathematics, Wiley-Interscience, New York, 2000.

[20] Z. Dong and T.G. Xu, One-dimensional stochastic Burgers equation driven by Lévy processess, Journal of Functional Analysis, 243, no. 2, 631-678, 2007.

[21] F. Flandoli, Dissipativity and invariant measures for stochastic Navier-Stokes equations with a generalised noise, NoDEA 1, 403-423 (1994)

[22] A. Friedman, Partial differential equations, Holt, Rinehart and Winston, Inc., New York-Montreal, Que.-London, 1969

[23] J.M. Ghidaglia, A note on the strong convergence towards attractors of damped forced KdV equations, J. Differential Equations 110, no. 2, 356-359 (1994)

[24] E. Hausenblas and J. Seidler, Stochastic convolutions driven by martingales: maximal inequalities and exponential integrability. Stoch. Anal. Appl. 26, no. 1, 98-119 (2008)

[25] E. Hausenblas and J. Seidler, A note on maximal inequality for stochastic convolutions, Czechoslovak Math. J. 51(126), no. 4, 785-790 (2001)

[26] J. Hoffmann-Jrgensen and G. Pisier, The law of large numbers and the central limit theorem in Banach spaces, Ann. Probability 4, no. 4, 587-599 (1976) 
[27] O. Kallenberg, Foundations of modern probability. Second edition. Probability and its Applications. Springer-Verlag, New York, 2002.

[28] S. Kwapień, W. Woyczyński, Random SERIES ANd Stochastic Integrals: SINGLE AND MUltiple, Probability and its Applications, Birkhuser Boston, Inc., Boston, MA, 1992.

[29] S. Kwapień, M.B. Marcus and J. Rosiński, Two results on continuity and boundedness of stochastic convolutions, Ann. Inst. H. Poincar Probab. Statist. 42, no. 5, 553-566 (2006)

[30] A.E. Kyprianou, Introductory lectures on fluCtuations of Lévy processes with Applications, Universitext, Springer-Verlag, Berlin, 2006.

[31] P. Lescot, M. Röckner, Perturbations of generalized Mehler semigroups and applications to stochastic heat equations with Lévy noise and singular drift, Potential Anal. 20, no. 4, 317-344 (2004)

[32] W. Linde, Probability in Banach spaces - stable and infinitely divisible DistribuTIONS, 2ND ED. A Wiley-Interscience Publication, 1986.

[33] J.-L. Lions and E. Magenes. Non-homogeneous boundary value problems and applications. Vol. I. Springer-Verlag, New York, 1972. Translated from the French by P. Kenneth, Die Grundlehren der mathematischen Wissenschaften, Band 181.

[34] A. Lunardi, Analytic semigroups and optimal Regularity in parabolic problems, Progress in Nonlinear Differential Equations and their Applications, 16, Birkhäuser Verlag, Basel, 1995.

[35] W. McLean. Strongly elliptic systems and boundary integral equations. Cambridge University Press, Cambridge, 2000.

A note on maximal inequality for stochastic convolutions, Czechoslovak Math. J. 51(126),

[36] C. Mueller, The heat equationwith Lévy noise, Stochastic Processes and Applications 74, 67-82, 1998.

[37] L. Mytnik, Stochastic partial differential equation driven by stable noise, Probability Theory and related Fields, 123, 157-201, 2002.

[38] A. Pazy. Semigroups of Linear Operators and Applications to Partial Differential Equations. Springer-Verlag, New York, 1983.

[39] S. Peszat and J. Zabczyk, Stochastic heat and wave equations driven by an impulsive noise, in Stochastic Partial Differential Equations and Applications VII, eds. G. Da Prato, and L. Tubaro, Lecture Notes in Pure and Applied mathematics, 245, 229-242, 2006.

[40] S.Peszat and J. Zabczyk, Stochastic Partial Differential Equations with Lévy NoIsE. An Evolution Equation Approach. Encyclopedia of Mathematics and its Applications 113, Cambridge University Press, Cambridge, 2007

[41] Pisier, G., Probabilistic Methods in the Geometry of Banach Spaces, in Probability and Analysis (Varenna 1985) pp. 167-241, LNM 1206, Springer Verlag, Berlin Heidelberg New York, 1986.

[42] R. Rosa, The global attractor for the 2D Navier-Stokes flow on some unbounded domains, Nonlinear Analysis, 32, 71-85 (1998)

[43] K. Sato, LÉvy processes and infinitely divisible distributions, Cambridge Studies in Advanced Mathematics, 68, Cambridge University Press, Cambridge, 1999.

[44] R. Seeley, Interpolation in $L^{p}$ with boundary conditions, Studia Math. 44, 47-60; Collection of articles honoring the completion by Antoni Zygmund of 50 years of scientific activity, I (1972)

[45] H. Tanabe, Equations of Evolution, Monographs and Studies in Mathematics, vol. 6, Pitman (Advanced Publishing Program), Boston, Mass., 1979.

[46] H. Tanabe, Functional analytic methods for partial differential equations, Monographs and Textbooks in Pure and Applied Mathematics, vol. 204, Marcel Dekker Inc., New York, 1997.

[47] M. E. Taylor. Pseudodifferential Operators. Princeton University Press. Princeton, New Jersey, 1981.

[48] H. Triebel. Theory of function spaces, volume 78 of Monographs in Mathematics. Birkhäuser Verlag, Basel, 1983. 
[49] H. Triebel. Interpolation theory, function spaces, differential operators. Johann Ambrosius Barth, Heidelberg, second edition, 1995.

[50] A. Truman and J.L. Wu, Fractional Burgers equation driven by Levy noise, in Stochastic Partial Differential Equations and Applications VII, eds. G. Da Prato, and L. Tubaro, Lecture Notes in Pure and Applied mathematics, 245, 293-310, 2006.

[51] M. Veraar, Non-autonomous stochastic evolution equations and applications to stochastic partial differential equations, preprint arXiv:0806.4439

[52] A. Yagi, Abstract quasilinear evolution equations of parabolic type in Banach spaces, Boll. Un. Mat. Ital. B (7) 5, no. 2, 341-368 (1991)

Department of Mathematics, The University of York, Heslington, York YO10 5DD, UK

E-mail address: zb500@york.ac.uk

Institute of Mathematics, Polish Academy of Sciences, P-00-950 Warszawa, Poland E-mail address: zabczyk@panim.impan.gov.pl 